\title{
Possible reasons for underestimating Joule heating in global models: $E$ field variability, spatial resolution, and vertical velocity
}

\author{
Yue Deng ${ }^{1,2}$ and Aaron J. Ridley ${ }^{2}$ \\ Received 13 August 2006; revised 6 June 2007; accepted 22 June 2007; published 28 September 2007.
}

[1] It is important to understand Joule heating because it can significantly change the temperature structure, atmosphere composition, and electron density and hence influences satellite drag. It is thought that many coupled ionosphere-thermosphere models underestimate Joule heating because the spatial and temporal variability of the ionospheric electric field is not totally captured within global models. Using the Global Ionosphere Thermosphere Model (GITM), we explore the effect of the electric field temporal variability, model resolution, and vertical velocity differences between ion and neutral flows on Joule heating in a self-consistent thermosphere/ionosphere system. First, the response of Joule heating to a step change in the externally driven electric field has been studied. While Joule heating is strongly affected by the convection electric field, both neutral winds and electron densities can significantly alter the spatial distribution of the Joule heating. Owing to the ramping up of neutral winds, there is a temporal variation of the Joule heating energy deposition rate when the electric field is constant. Second, we compare the calculated neutral gas heating rates when GITM is run with three different temporal variations of the electric fields, having the same temporally averaged electric field $(\bar{E})$ but different standard deviations $\left(\sigma_{E}\right)$. The neutral gas heating rate increases with the electric field temporal variability, and due to the feedback of the neutral winds and electron densities, the percentage increase is different from $\sigma_{E}^{2} / \bar{E}^{2}$, which is normally used to describe the effect of electric field temporal variability on the Joule heating. Third, comparison of the neutral gas heating rate with different model resolutions shows that at $200 \mathrm{~km}$ altitude, the polar average neutral gas heating rate increases by $20 \%$ when the latitudinal resolution increases from $5^{\circ}$ to $1.25^{\circ}$. This is due to the model's ability to better capture small-scale features in the electric field and particle precipitation. Last, inclusion of the vertical velocity difference (which is neglected in many models) is less significant than the other two factors and appears to be negligible at high latitudes. While the magnitude of the neutral gas heating rate at middle and low latitudes is smaller than that at high latitudes, the relative importance of the vertical velocity difference is larger, and the contribution can reach $15 \%$ of the averaged Joule heating at middle and low latitudes.

Citation: Deng, Y., and A. J. Ridley (2007), Possible reasons for underestimating Joule heating in global models: $E$ field variability, spatial resolution, and vertical velocity, J. Geophys. Res., 112, A09308, doi:10.1029/2006JA012006.

\section{Introduction}

[2] High-latitude Joule heating is one of the most significant energy deposition processes from the magnetosphere into the ionosphere-thermosphere system. During the January 1997 magnetic cloud event, 47\% of the solar wind energy was deposited in the form of Joule heating, while

\footnotetext{
${ }^{1}$ High Altitude Observatory, National Center for Atmospheric Research, Boulder, Colorado, USA.

${ }^{2}$ Center for Space Environment Modeling, University of Michigan, Ann Arbor, Michigan, USA.

Copyright 2007 by the American Geophysical Union. 0148-0227/07/2006JA012006
}

$22 \%$ was in the form of particle heating [ $\mathrm{Lu}$ et al., 1998]. During a typical storm, more than half of the energy is deposited through Joule heating [Sharber et al., 1988]. Joule heating has significant consequences in the thermosphere and ionosphere. The obvious response is the rapid increase of temperature, which causes upwelling of the neutral atmosphere [Prölss et al., 1991; Fuller-Rowell et al., 1994; Lühr et al., 2004; Liu and Lühr, 2005] and a subsequent increase of the atmospheric drag on satellites. In addition, the accompanying traveling atmospheric disturbances and largescale storm circulation can cause global ionospheric storm effects [Fuller-Rowell et al., 1994; Prölss et al., 1991].

[3] While Joule heating has been investigated utilizing measurements obtained by satellites [Rich et al., 1987; 
Heelis and Coley, 1988; Kelley et al., 1991; Gary et al., 1995; Lühr et al., 2004] and ground-based radars, [Banks et al., 1981; Kamide and Kroehl, 1987; de La Beaujardiére et al., 1991; Thayer et al., 1995; Thayer, 1998], it is currently impossible for observations to give a precise specification of global Joule heating due to the difficulty of observing conductivity, electric field, and neutral wind simultaneously at all locations. Furthermore, these contributing variables respond independently to specific sources of energy. Coupled global ionosphere-thermosphere models provide a framework to study these variables and offer the ability to investigate the three-dimensional (3-D) distribution of Joule heating. However, many global models have had a difficult time modeling Joule heating accurately [Codrescu et al., 1995]. Joule heating has been consistently underestimated because it is frequently assumed in general circulation models (GCMs) that the electric field is relatively smooth both in space and time. Codrescu et al. [1995] showed that the polar region electric field is variable and that the variability can significantly increase the amount of Joule heating. Since then, many studies have investigated the characteristic of electric field temporal variability. For example, using the Millstone Hill ion drift observations, Codrescu et al. [2000] examined the electric field model patterns and the associated variability for different geomagnetic conditions and seasons. Crowley and Hackert [2001] used the Assimilative Mapping of Ionospheric Electrodynamics (AMIE) [Richmond, 1992] procedure to characterize electric field temporal variability. Matsuo et al. [2003] investigated the dependence of electric field variability on IMF and dipole tilt by analyzing Dynamics Explorer 2 (DE-2) measurements. Using the satellite CHAMP data, Lühr et al. [2004] analyzed the correlation between fieldaligned currents (FAC) and the neutral density enhancements in the cusp region. They found that small-scale FAC filaments are significant to Joule heating.

[4] Electric field spatial variability, as well as temporal variability, strongly affect the accurate calculation of Joule heating. Using data from the Dynamics Explorer 2 (DE2) satellite, Kivanc and Heelis [1998] and Johnson and Heelis [2005] investigated the observed ion velocity structure in the $F$ region. Johnson and Heelis [2005] found the enhancement in Joule heating from the presence of spatial structure below $1.28^{\circ}$ could be $10 \%$, due to the electric field spatial variability.

[5] For global simulations, the challenge of examining the effects of spatial variability of the electric field on Joule heating comes from two factors: (1) the resolution of the general circulation models is around $2^{\circ}-5^{\circ}$ latitude (Coupled Thermosphere-Ionosphere-Plasmasphere model, CTIP, and Thermosphere-Ionosphere-Electrodynamics General Circulation Model, TIEGCM, respectively) and is therefore not high enough to catch the small-scale structure of the electric field (or auroral precipitation) and plasma density; and (2) the ability of the electrodynamic models [e.g., Richmond and Kamide, 1988; Weimer, 1996] to accurately describe the electric field spatial variability, which depends on the type and resolution of the electrodynamic model. The averaged and smoothed climatologies can only give poor spatial variability, since averaging massive amounts of data smears out extremes, which may, in fact, contribute significant amounts to the local, and possibly global, Joule heating.

[6] Besides the electric field variability, several other factors are also important in determining Joule heating. Thayer et al. [1995] showed that the neglect of the neutral dynamics in Joule heating calculations can be misleading. Lu et al. [1995b] documented that the neutral winds could reduce Joule heating by $28 \%$. The ionospheric electron density also plays an essential role in determining the Joule heating rate. Without an accompanying level of ionospheric density, large electric fields may not result in large neutral gas heating rates. Because of positive and negative storm effects, the Joule heating may be significantly different than expected when using specified conductances. Using a selfconsistent thermosphere/ionosphere model, all of these features can be captured, and the studies of the electric field spatial and temporal variability can be conducted in a much more realistic manner.

[7] While some studies have been done to examine the neutral wind and conductance effect on Joule heating, few studies have been conducted in a self-consistent thermosphere/ionosphere system using simple tests to quantify the effects of the electric field variability. Studies such as Codrescu et al. [2000], Crowley and Hackert [2001], Matsuo et al. [2003], and Johnson and Heelis [2005] simply examine the contribution of the temporal and spatial variability in an idealized environment, where the conductance is specified and the neutral winds are neglected. In this study all of the feedback and couplings have been included through using the self-consistent Global Ionosphere Thermosphere Model (GITM), a newly created model that is ideally suited to this type of study. For example, one important factor to consider, when electric field variability is examined, is the time step of the model and the time resolution of the electric field. Models such as the TIEGCM have time steps of $5 \mathrm{~min}$, while CTIP updates its electric field every $12 \mathrm{~min}$. Ridley et al. [1997] showed that the average time for the ionospheric electric field to change is $12 \mathrm{~min}$. Therefore models that do not update their electric fields more often than about 3 min are missing a significant amount of the temporal variability. GITM can update its electric field approximately every $2 \mathrm{~s}$ but typically does it every minute.

[8] Resolution of the models is another obvious consideration when examining the effects of spatial variability. Since most GCMs are using fixed (low) resolution, it is impossible for them to investigate the impact of smallerscale structures on Joule heating. One advantage of GITM, compared with other models of the thermosphere and ionosphere, is its flexible grid resolution. This characteristic of GITM makes it possible to investigate the importance of spatial resolution in modeling Joule heating. This is important because the community needs to understand and quantify the amount of Joule heating that is missed due to the low resolution in typical simulations.

[9] In addition to the above considerations, the vertical component of the flows have typically been neglected when Joule heating is calculated in many GCMs because only the relative difference between the horizontal ion drift and the horizontal neutral wind is taken into account in the Joule heating calculation [Killeen and Roble, 1984; Roble et al., 


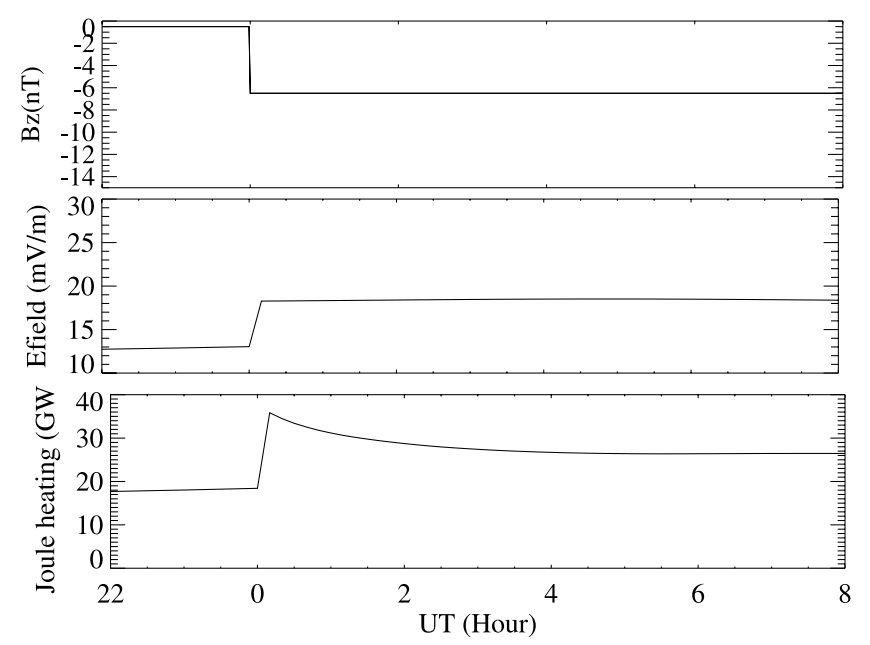

Figure 1. From top to bottom: Temporal variation of IMF $B_{z}$, polar average (poleward of $60^{\circ}$ ) electric field and hemispherically integrated (poleward of $60^{\circ}$ ) Joule heating energy deposition rate during 2200-0800 UT.

1988]. However, the vertical ion flow can reach hundreds of $\mathrm{m} / \mathrm{s}$ [Deng and Ridley, 2006b] and the vertical neutral wind can exceed $50 \mathrm{~m} / \mathrm{s}$. While the approximation of no vertical component contribution may be acceptable, it should, at least, be quantified. Further, at low latitudes, where the vertical component of the flows can be more significant (due to vertical $\mathbf{E} \times \mathbf{B}$ drifts), Joule heating due to the vertical flows may be nonnegligible.

\section{Model Description and Simulation Conditions}

[10] GITM is a three-dimensional spherical code that models the Earth's thermosphere and ionosphere system using a stretched grid in latitude and altitude [Ridley et al., 2006]. In addition, the number of grid points in each direction can be specified, so the resolution is extremely flexible. GITM is different than other global ionosphere thermosphere models in that it relaxes the hydrostatic equilibrium condition on the thermosphere, allowing significant vertical winds to form due to nongravitational forces such as ion drag, coriolis, and centrifugal acceleration. The primary tradeoff is the time step, which is close to $2 \mathrm{~s}$ in GITM and much smaller than that in hydrostatic model $(3-5 \mathrm{~min})$. This is caused by the need to resolve the sound waves in the thermosphere in GITM, and the small time step influences the model simulating speed. The primary equations (specified by Ridley et al. [2006]) are listed in Appendix A for completeness.

[11] GITM can use a dipole or the international geophysical reference field (IGRF) magnetic field with the APEX coordinate system [Richmond, 1995]. This allows experiments ranging from highly idealized magnetic field topological cases to realistic magnetic field cases. It can be coupled to a large number of models of high-latitude ionospheric electrodynamics, such as the AMIE technique [Richmond and Kamide, 1988; Richmond, 1992] in realistic, highly dynamic time periods, or Weimer [1996], Foster [1983], Heppner and Maynard [1987], or Ridley et al. [2000] electrodynamic potential patterns and the Hardy et al. [1987] or Fuller-Rowell and Evans [1987] particle precipitation patterns for more idealized conditions. GITM is also part of the University of Michigan's Space Weather Modeling Framework [Tóth et al., 2005], so it can be coupled with a global magnetohydrodynamic (MHD) model [Powell et al., 1999] of the magnetosphere. This allows investigation of the coupling between the thermosphereionosphere and the magnetosphere systems [e.g., Ridley et $a l ., 2003]$. GITM was compared with MSIS and IRI to show that the large-scale features are reproduced with the code [Ridley et al., 2006], while Deng and Ridley [2006a] showed the consistency of simulated neutral wind with the observation from the Wind Imaging Interferometer (WINDII) instrument and did a statistical validation of the neutral winds within the model.

[12] The simulations described in this study started from MSIS [Hedin, 1983] and IRI [Rawer et al., 1978], with static neutrals and a dipole magnetic field. The Weimer [1996] electrodynamic potential patterns and the FullerRowell and Evans [1987] particle precipitation patterns are used as high-latitude drivers. Since the high-latitude potential and particle precipitation are specified from external electrodynamic drivers for this study, the coupling to the magnetosphere is not self-consistent. Without special declaration, the model resolution is $2.5^{\circ}$ latitude by $5^{\circ}$ longitude by approximately $1 / 3$ scale height. The initial conditions are equinox, moderate solar activity $\left(F_{10.7}=150\right)$, and quiet geomagnetic activity (Hemisphere Power $=10 \mathrm{GW}$, Interplanetary Magnetic Field $B_{y}=0 \mathrm{nT}$ and $B_{z}=-0.5 \mathrm{nT}$ ). GITM is run for 24 hours using these drivers to allow the system to evolve to a quasi-steady state solution before conducting the simulation described below.

\section{Results}

\subsection{Response of Joule Heating to a Step Change of Electric Field}

[13] In order to examine the thermospheric reaction to a simple step change of the high-latitude electric field, the magnitude of the southward component of the interplanetary magnetic field (IMF) $B_{z}$ is increased from 0.5 to $6.5 \mathrm{nT}$, without changing other inputs (such as the hemispheric power), after running GITM for 24 hours to reach a quasi-steady state. The IMF is used to specify the electric field change, since it is the primary driver for almost all modern empirical models of the ionospheric potential. The cross polar cap potential (CPCP) rises from 40 to $75 \mathrm{kV}$, and the polar average electric field magnitude correspondingly increases from 13 to $19 \mathrm{mV} / \mathrm{m}$ when $B_{z}$ changes, as shown in Figure 1. Because $B_{z}$ does not change during 00000800 UT, and Weimer [1996] does not take into account the contribution of the neutral wind to the polar cap potential, the average electric field is almost constant from 0000 to 0800 UT. The electric field is not exactly constant due to a very slight UT dependence of the electric field in the Weimer [1996] model. This is a result of the UT-dependence of dipole tilt, although in these simulations, the dipole tilt is constant at zero degrees. This variation is less than $2 \%$.

[14] Joule heating above $60^{\circ}$ north latitude is integrated and is shown in Figure 1c. The Northern Hemisphere integrated value rises from 18 to $37 \mathrm{GW}$ after the electric field change at high latitudes. After the initial rise, the 
(A) Electric field $(m V / m)$

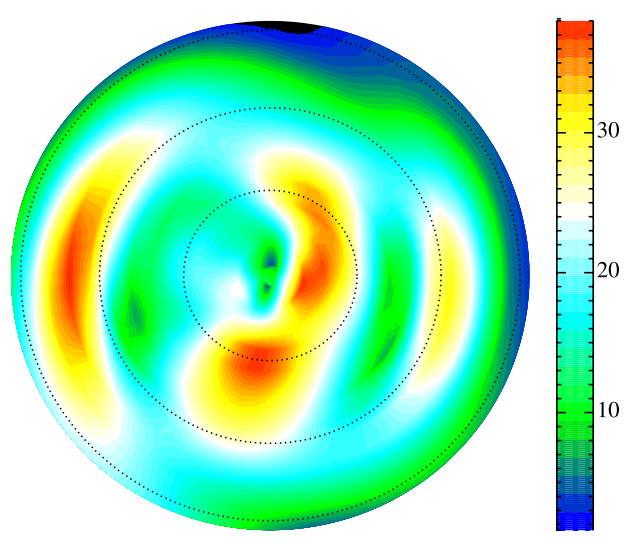

(B)Height-integrated Joule heating energy

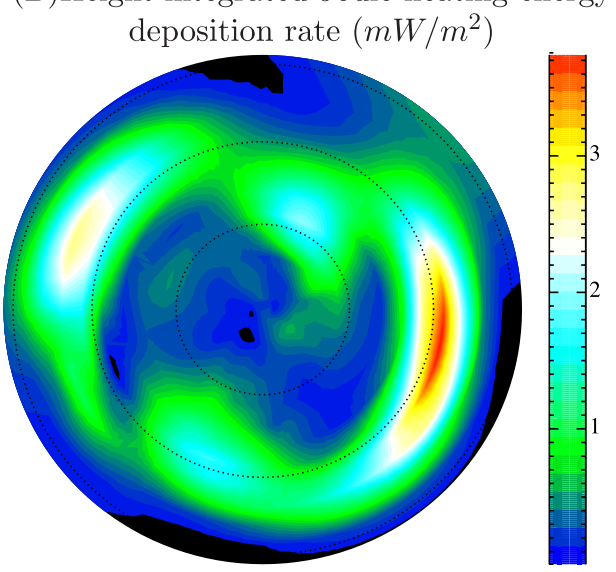

Figure 2. Polar distribution of temporally averaged (0000-0800 UT) of (a) electric field and (b) heightintegrated Joule heating energy deposition rate. Noon is on the top, midnight is at the bottom, while dawn is on the right. The outside ring is $60^{\circ}$.

integrated Joule heating energy deposition rate decreases approximately $20 \%$, indicating the influence of other factors besides the electric fields (such as the neutral winds) in the Joule heating calculation. This decrease is one of the main purposes of studying the effects of the electric field variability on Joule heating using a self-consistent thermosphere/ionosphere model instead of just examining the electric field and speculating how the Joule heating is effected. This reaction in the self-consistent thermosphere/ ionosphere system will be discussed in more detail in section 3.1.1.

[15] Figure 2 shows the electric field and height-integrated Joule heating energy deposition rate spatial distributions averaged over the time period from 0000 to 0800 UT. The electric field shows a triple peak distribution, with enhancements at dawn, dusk, and within the polar cap. The maximum Joule heating energy deposition rate occurs near dawn and has a value close to $3.8 \mathrm{~mW} / \mathrm{m}^{2}$. The distribution and magnitude are very consistent with the result in the work of Thayer et al. [1995], which is also representative of moderate to quiet geomagnetic activity (HP index $=11 \mathrm{GW}$, $\mathrm{CPCP}=75 \mathrm{kV}$, and $F_{10.7}=220$ ). Our result misses the maximum value in the midnight auroral region due to the lack of substorms in the Weimer [1996] model, and a lack of change in the hemispheric power in our idealized simulation. The conditions described above are similar to what may be observed during steady magnetospheric convection events [e.g., DeJong and Clauer, 2005].

\subsubsection{Influence of Neutral Wind and Electron Density on Joule Heating}

[16] As shown in Figure 2, the spatial distributions of the electric field magnitude and the neutral gas heating rate caused by Joule heating show significant differences. While the electric field has three maxima: on the dawnside, on the duskside, and in the polar cap, the neutral gas heating rate only maximizes on the dawnside with a small peak near dusk. The dawn-dusk asymmetric pattern has also been shown in the work of Thayer et al. [1995], Lu et al. [1995b], and [McHarg et al. [2005], which is explained as the result of the influence of the neutral wind on the ionospheric electrodynamics and the influence of the electron density on the conductance. The physical mechanisms contributing to Joule heating are depicted in Figure 3. After the electric field increases, there are three ways to affect Joule heating. First, the electric field is a dominant factor and can directly change Joule heating by varying the ion convection. Second, since Joule heating is proportional to the difference of neutral and ion flows, the neutral wind can strongly affect both the temporal variation and spatial distribution of Joule heating. Finally, another feedback mechanism is from the change of the electron density, which is subject to the variation of the electric field and neutral density as shown in Figure 3. The enhanced electric field redistributes the electron density in the polar region, for example, extending the tongue of ionization, stretching the trough at lower latitudes and vertically shifting the plasma [Sojka and Schunk, 1989; Deng and Ridley, 2006b]. The effect of the electric field on the electron density is quite dependent on the position and can both increase and decrease the density. In addition, the increased Joule heating raises the neutral

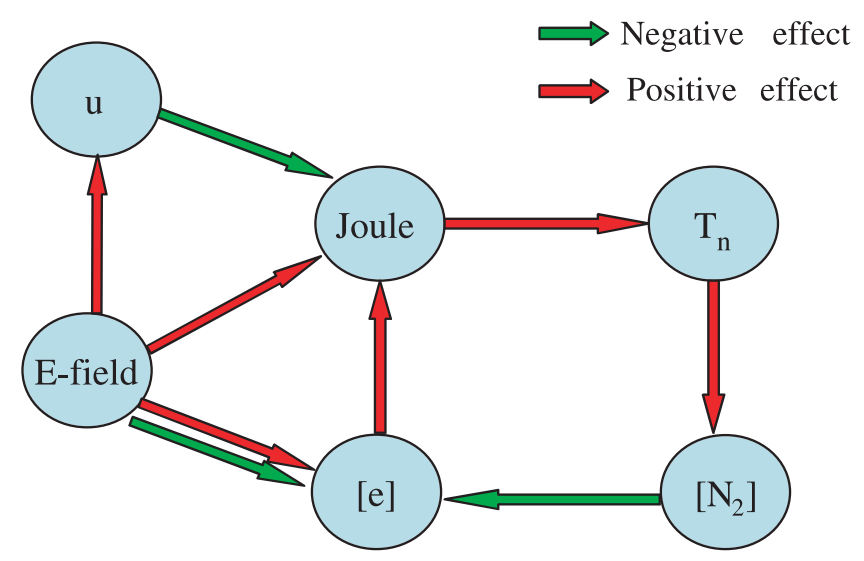

Figure 3. Diagram of the relationship between difference parameters. After the electric field changes, both neutral wind and electron density, as well as the electric field, can strongly affect Joule heating. 

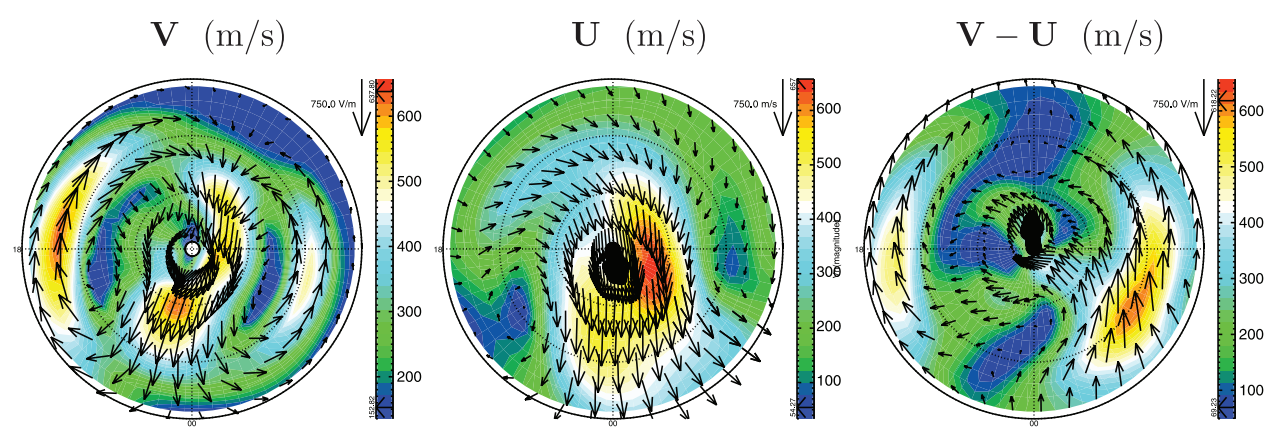

Figure 4. The distributions of ion convection (left), neutral wind (middle) and the difference between them (right) at $400 \mathrm{~km}$ altitude, 0400 UT. The colors (and length of arrows) show the speed and the arrows indicate the direction.

temperature, which increases the molecular density at high altitudes. This enhanced molecular density reduces the electron density through recombination. Consequently, the changed electron density varies the conductivity and also the Joule heating. The influence of the electric field on Joule heating is discussed further in section 3.2. Therefore in this section, influence of the other two less investigated factors, neutral winds and electron density distribution, are investigated.

[17] In order to estimate the importance of the neutral wind on Joule heating, the distributions of the ion velocity $(\mathbf{V})$, neutral wind $(\mathbf{U})$ and the difference between them $\mathbf{V}-\mathbf{U}$ are shown in Figure 4. The distribution of the ion velocity is a well-organized two-cell convection pattern. Owing to the combination of the ion drag force and the Coriolis effect, the neutral wind strongly follows the ion convection in the dusk region, while it weakly follows it in the dawn region [Killeen and Roble, 1984; Deng and Ridley, 2006a]. There- fore the difference between the ion and neutral flows is larger on the dawnside than on the duskside. These results are consistent with Thayer et al. [1995, Plate 4], which showed that the neutral wind reduces the height-integrated Joule heating energy deposition rate on the dusk cell and maximizes it on the dawn cell.

[18] Figure 5 shows that the neutral gas heating rate due to Joule heating, which is proportional to the electron density and $|\mathbf{V}-\mathbf{U}|$, can have a different spatial distribution than $|\mathbf{V}-\mathbf{U}|$. In order to investigate this discrepancy, the temporally averaged (0000-0800 UT) $N_{e},|\mathbf{V}-\mathbf{U}|$, and heating rate at $120 \mathrm{~km}$ and $400 \mathrm{~km}$ altitudes are shown. At $400 \mathrm{~km}$ altitude, $N_{e}$ has a large tongue of ionization over the polar cap but no apparent enhancement in the auroral region. Since there is no significant difference in the electron density between the dawnside and duskside at this altitude, the spatial distribution of the neutral gas heating rate is very similar to the spatial distribution of $|\mathbf{V}-\mathbf{U}|$. At
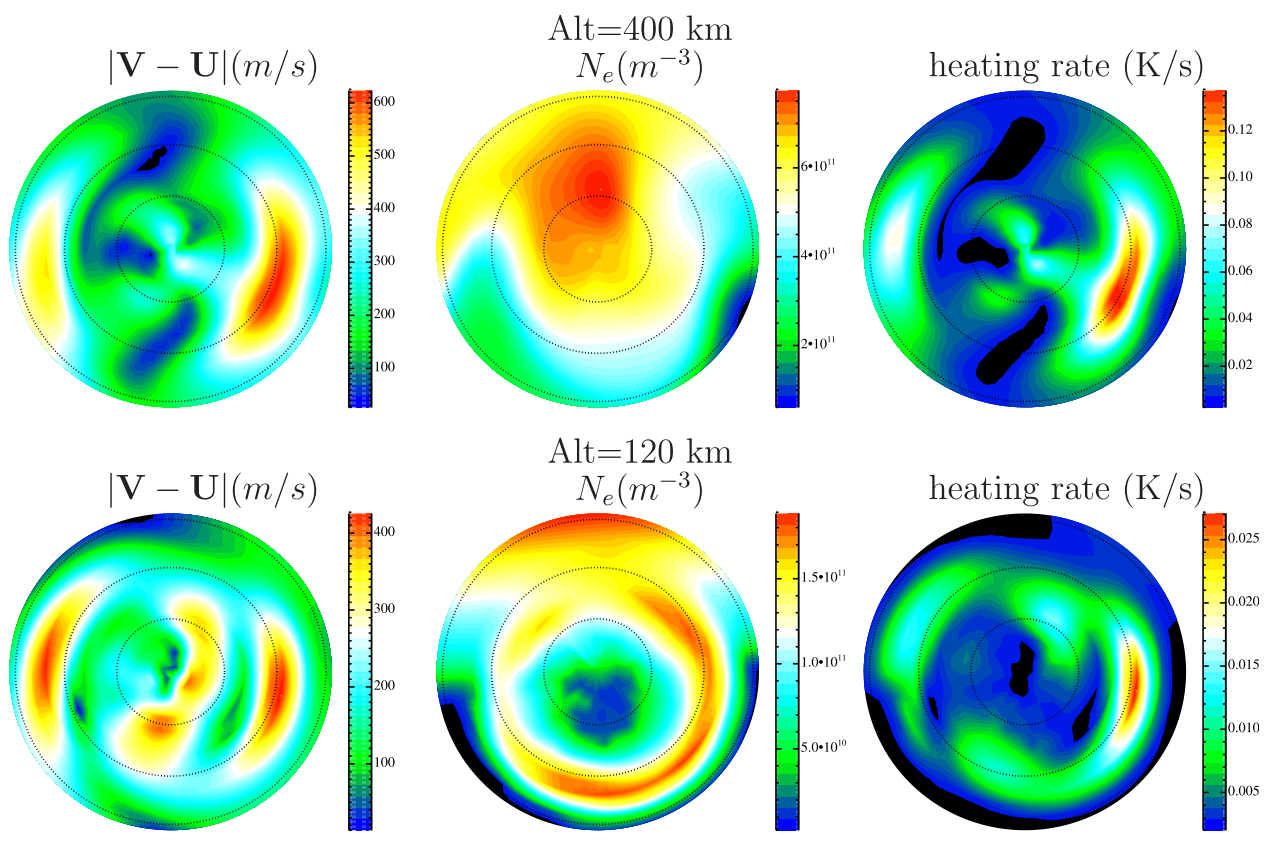

Figure 5. (top) Eight hour (0000-0800 UT) average $|\mathbf{V}-\mathbf{U}|$ (left), electron density (middle) and neutral gas heating rate caused by Joule heating (right) at $400 \mathrm{~km}$ altitude in the same format as Figure 4; (bottom) The same as the top except at $120 \mathrm{~km}$ altitude. 


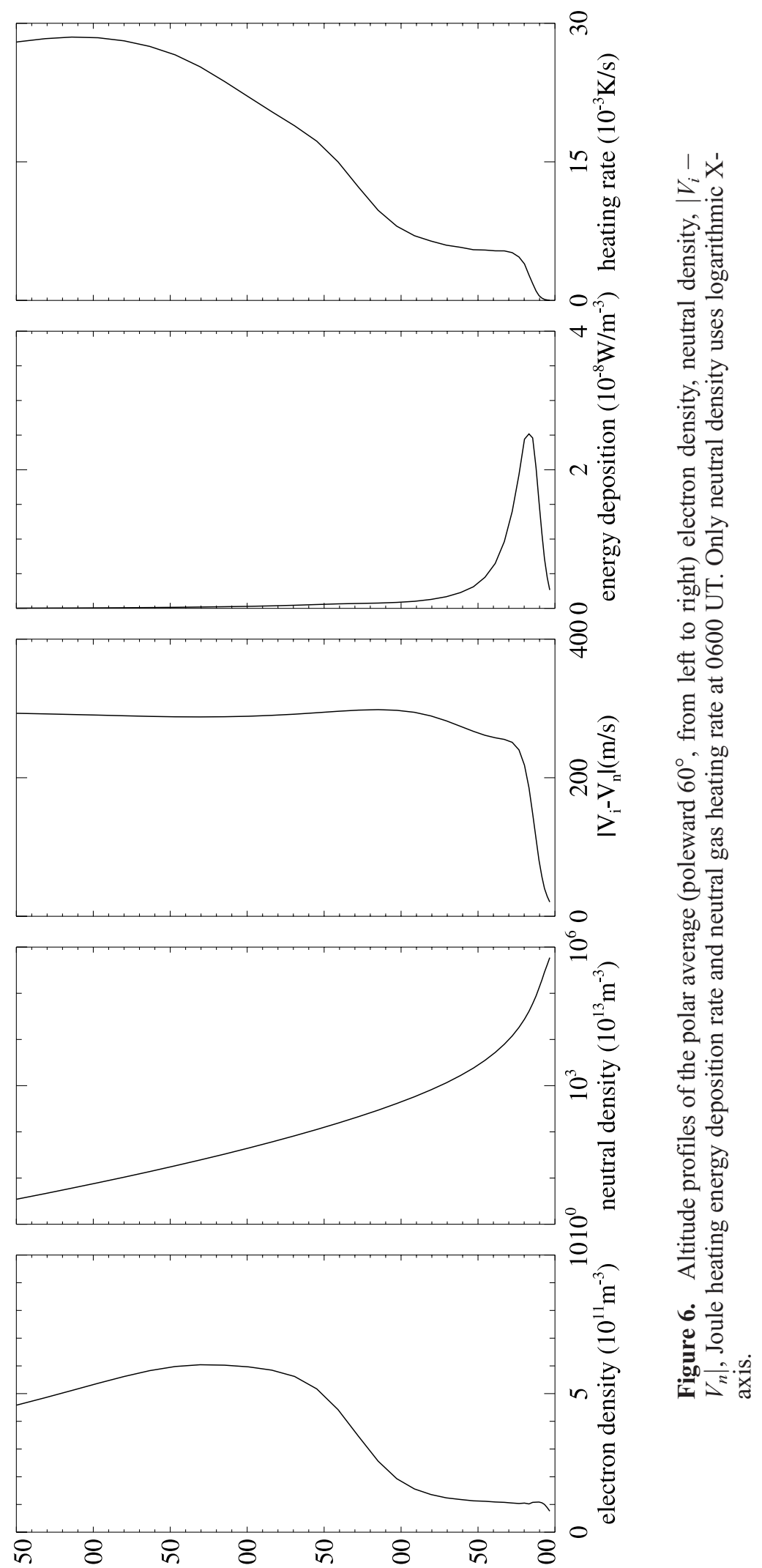




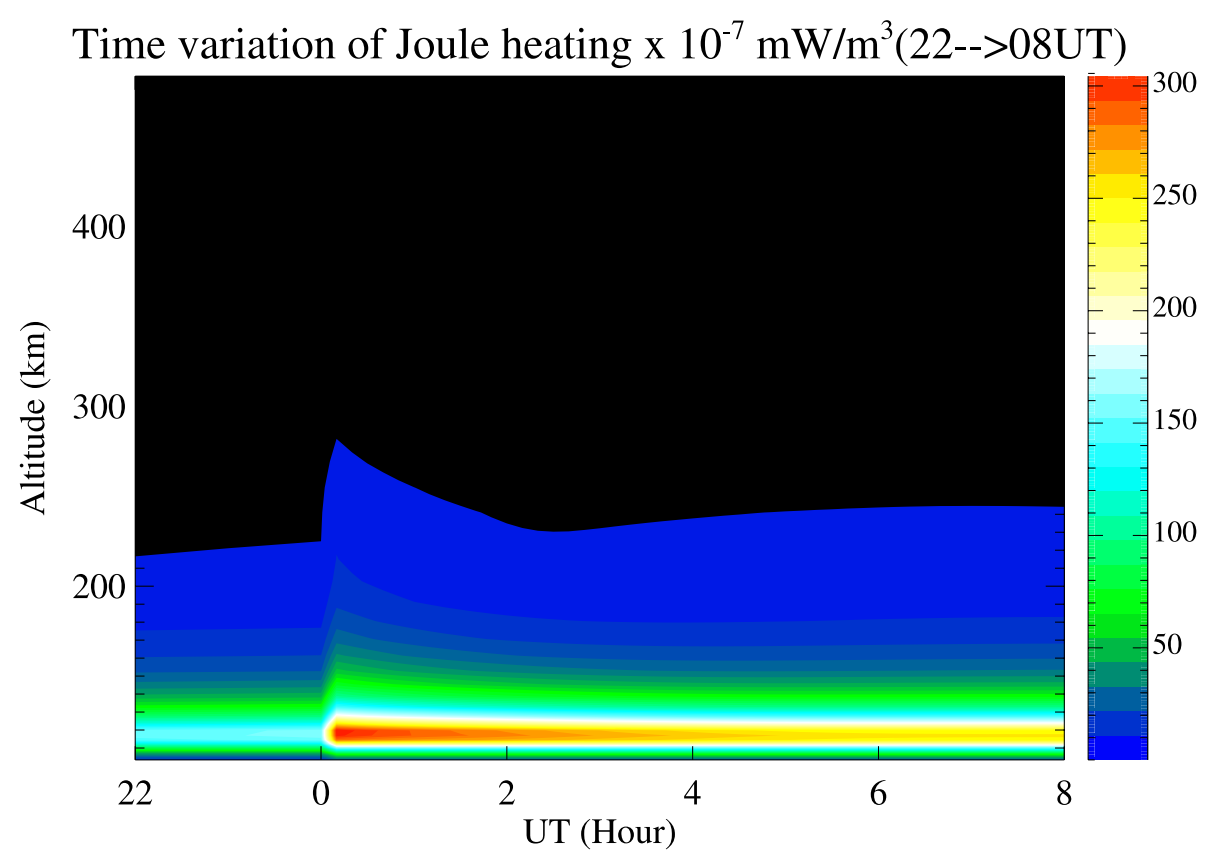

Figure 7. The temporal variation of the altitude profile of the polar average (poleward of $60^{\circ}$ ) Joule heating energy deposition rate.

$120 \mathrm{~km}$ altitude, as a consequence of particle precipitation, there is strong ionization in the auroral zone and the maximum electron density occurs in this region. While the dawnside and duskside peaks of $|\mathbf{V}-\mathbf{U}|$ are comparable (at $120 \mathrm{~km}$ ), the $N_{e}$ at the $|\mathbf{V}-\mathbf{U}|$ dawnside peak position is almost one order of magnitude larger than that at the $|\mathbf{V}-\mathbf{U}|$ duskside peak location. Consequently, the neutral gas heating rate is much larger on the dawnside. It is clear that the distribution of the electron density, as well as the electric field and the neutral wind, can significantly influence the neutral gas heating rate caused by Joule heating and that this is strongly dependent on altitude.

\subsubsection{Joule Heating Energy Deposition Rate Versus Neutral Gas Heating Rate}

[19] From equation (A7) in Appendix A, the Joule heating (friction heating to the thermosphere) energy deposition rate $\left(Q_{J}\right)$ is

$$
Q_{J}=N_{e} \bar{m}_{i} \nu_{\text {in }}(\mathbf{v}-\mathbf{u})^{2}
$$

where $N_{e}$ is electron number density, $\mathbf{V}$ is ion velocity, $\mathbf{U}$ is neutral velocity, and $\bar{m}_{i}$ is average ion mass. From the kinetic theory, the ion-neutral collision frequency is defined as $\nu_{i n}=C_{i n} N_{n}$, where $C_{i n}$ is a numerical coefficient and $N_{n}$ is neutral number density [Schunk and Nagy, 2000]. Equation (1) can therefore be written as:

$$
Q_{J}=\bar{m}_{i} C_{i n} N_{e} N_{n}(\mathbf{v}-\mathbf{u})^{2}
$$

The quantity $\bar{m}_{i} C_{i n}$ is almost constant with altitude, and therefore the Joule heating energy deposition rate is proportional to $N_{e} N_{n}(\mathbf{V}-\mathbf{U})^{2}$. As shown in Figure 6, as altitude increases, $N_{e}$ varies from $1 \times 10^{11}$ to $6 \times 10^{11} \mathrm{~m}^{-3}$, $N_{n}$ exponentially decreases, and $|\mathbf{V}-\mathbf{U}|$ is almost constant above $120 \mathrm{~km}$ altitude but decreases dramatically below
$120 \mathrm{~km}$. The combination of the three factors maximizes the Joule heating energy deposition rate around $120 \mathrm{~km}$ altitude.

[20] While the Joule heating energy deposition rate is the quantity that is typically discussed in Joule heating studies, it does not give a good indication of how the thermospheric temperature is actually changing. In order to examine that, the altitude profile of the neutral gas heating rate (i.e., the rate of change of temperature due to Joule heating) should be examined:

$$
R_{h}=\frac{Q_{J}}{\rho C_{v}}=\frac{Q_{J}}{\rho \frac{k}{m(\gamma-1)}}
$$

where $\rho$ is mass density, $C_{v}$ is heat capacity at constant volume. $C_{v}$ is used in GITM (instead of $C_{p}$ ) because the cells are constant in volume but not constant in pressure, unlike other models. Substituting equation (2) into equation (3):

$$
R_{h}=\frac{\gamma-1}{k} \bar{m}_{i} C_{i n} N_{e}(\mathbf{v}-\mathbf{u})^{2}
$$

Since $\frac{\gamma-1}{k} \bar{m}_{i} C_{i n}$ changes little with altitude, the neutral gas heating rate is proportional to $N_{e}(\mathbf{V}-\mathbf{U})^{2}$, such that $N_{n}$ has little impact on it. As shown in Figure 6, while $|\mathbf{V}-\mathbf{U}|$ is almost constant above $120 \mathrm{~km}$ altitude, there is a large $N_{e}$ peak in the $F_{2}$ layer. Therefore the neutral gas heating rate maximizes around $400 \mathrm{~km}$, which is significantly different from the altitude profile of the Joule heating energy deposition rate. Thayer and Semeter [2004] also showed a similar difference between the Joule heating energy deposition rate and the neutral gas heating rate.

[21] Figure 7 shows the temporal variation of the altitude profile of the polar-averaged Joule heating energy deposition rate from 2 hours before to 8 hours after the $B_{z}$ change. 


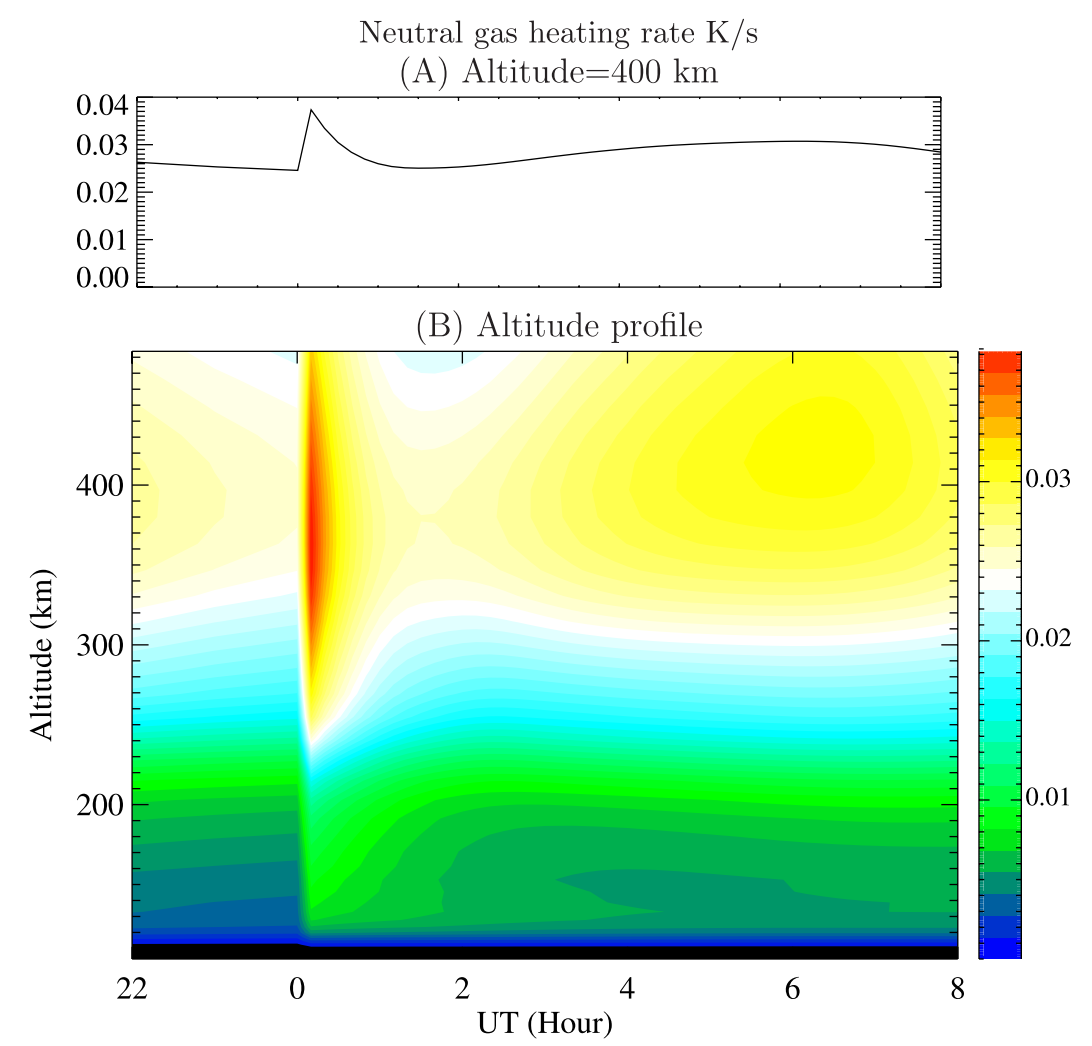

Figure 8. (a) The temporal variation of the polar-averaged (poleward of $60^{\circ}$ ) neutral gas heating rate at $400 \mathrm{~km}$ altitude. (b) Same as Figure 7, but for neutral gas heating rate.

(A) $|\mathbf{V}|,|\mathbf{U}|$ and $|\mathbf{V}-\mathbf{U}|$ at $400 \mathrm{~km}$ altitude
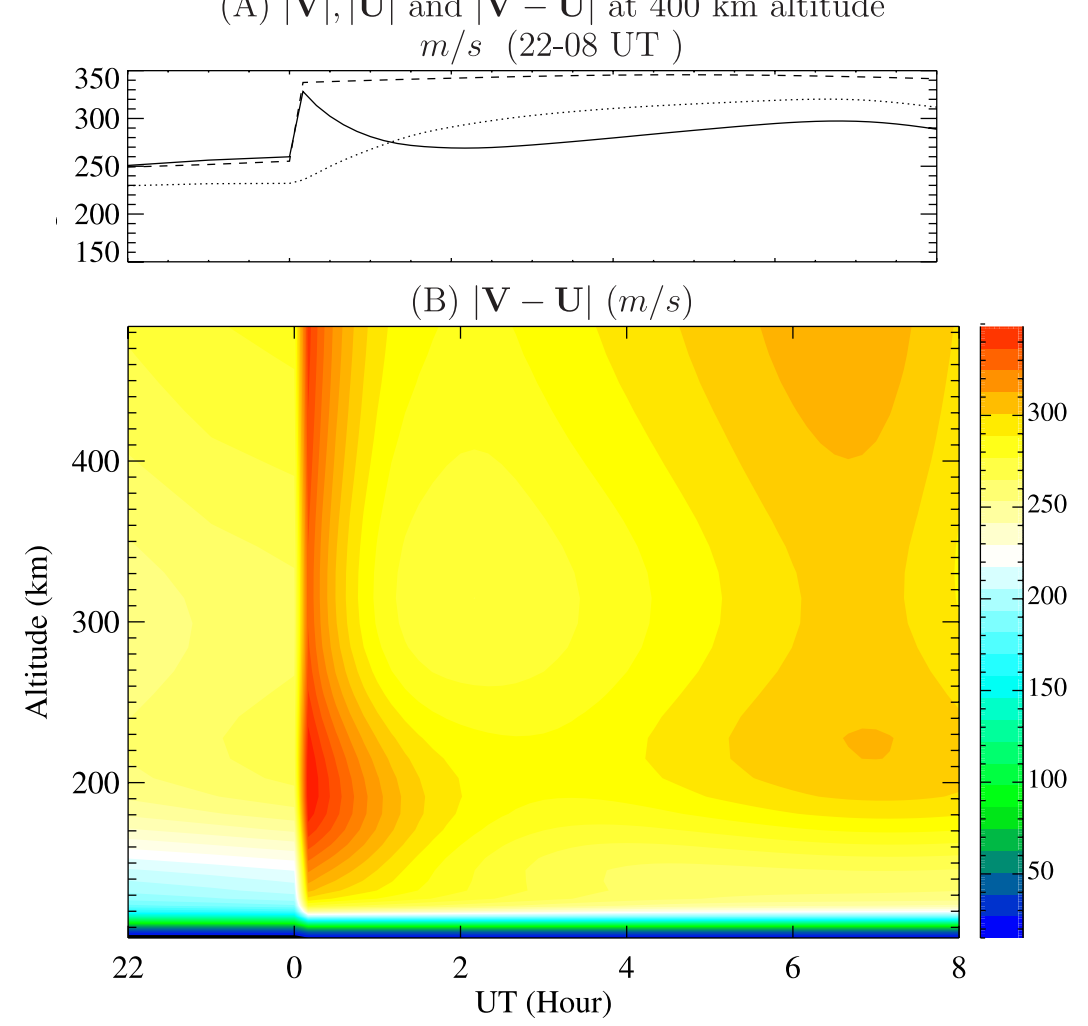

Figure 9. Same as Figure 8, but (a) for $|\mathbf{V}|$ (dash line), $|\mathbf{U}|$ (dot line) and $|\mathbf{V}-\mathbf{U}|$ (solid line); (b) for $|\mathbf{V}-\mathbf{U}|$. 


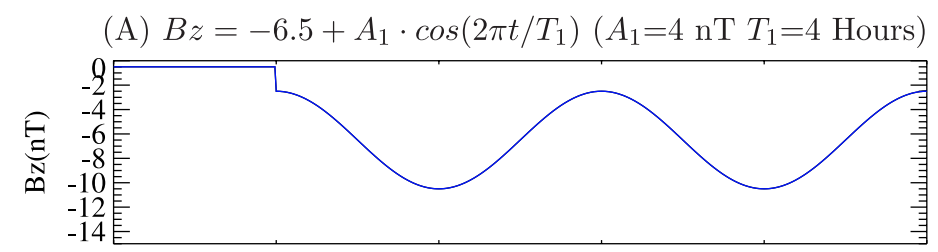

(B) neutral gas heating rate at $400 \mathrm{~km}$ altitude $(\mathrm{K} / \mathrm{s})$

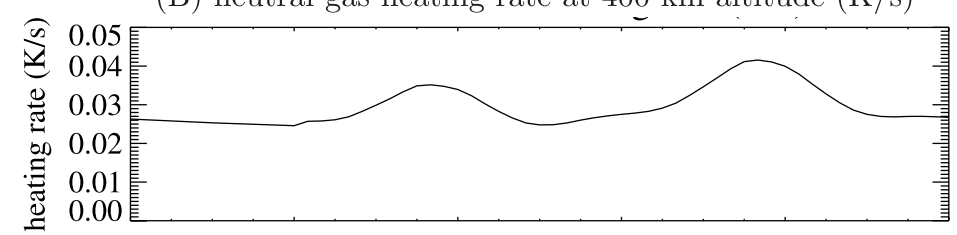

(C) Neutral gas heating rate $K / s$

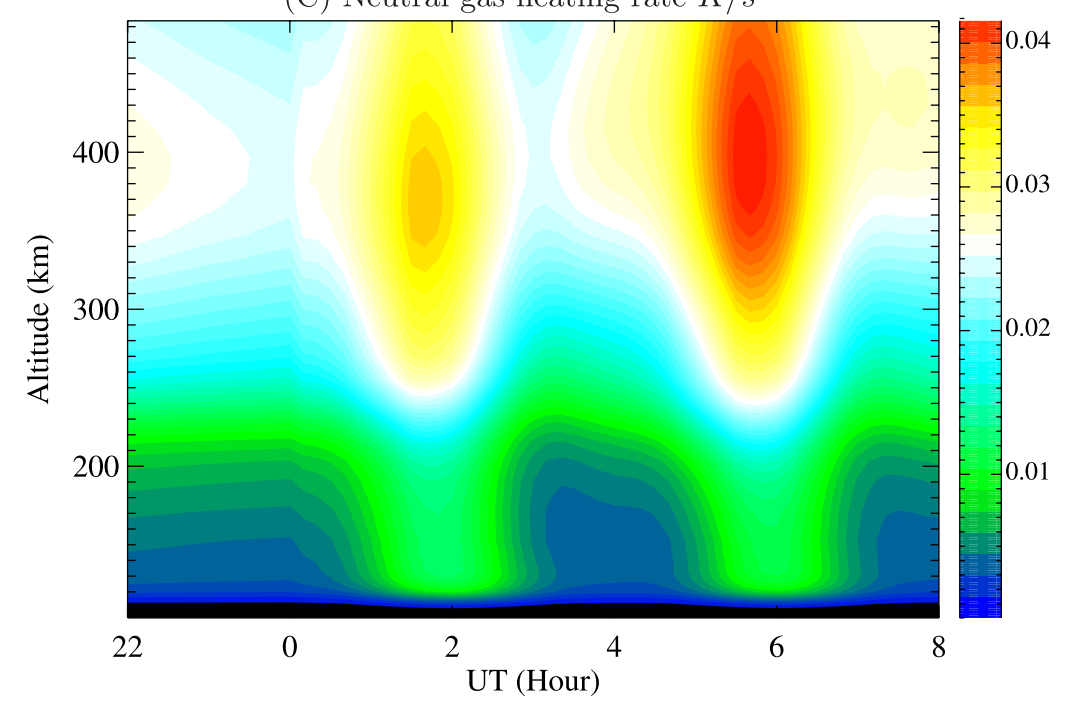

Figure 10. The temporal variation of (a) input (IMF $B_{z}$ ), (b) the resulting neutral gas heating rate at $400 \mathrm{~km}$ altitude, and (c) the resulting altitude profile of the polar-averaged (poleward of $60^{\circ}$ ) neutral gas heating rate.

Each point represents the polar-averaged Joule heating energy deposition rate northward of $60^{\circ}$ at the specified altitude. The rate increases by $70 \%$ abruptly after the change of electric field. In contrast, Figure 8 shows the temporal variation of the polar averaged neutral gas heating rate as a function of altitude and time. These figures show that the Joule heating energy deposition rate and the neutral gas heating rate have very different height profiles, with the neutral gas heating rate peaking at $400 \mathrm{~km}$ altitude instead of $120 \mathrm{~km}$. Since one of the primary concerns about Joule heating is its impact on the thermospheric temperature structure, the change of the neutral gas heating rate is investigated instead of the Joule heating energy deposition rate.

[22] As shown in Figure 8, there is a temporal variation of the neutral gas heating rate between 0000 UT and 0800 UT above $300 \mathrm{~km}$ altitude, which produces a secondary peak at $0630 \mathrm{UT}$ and $400 \mathrm{~km}$ altitude. It is quite interesting that this peak is $20 \%$ above the background level when the electric field is almost constant ( $2 \%$ variation). Figure 9 shows that $|\mathbf{V}-\mathbf{U}|$ varies at a similar frequency as the neutral gas heating rate and also has a second peak around 0630 UT. Since the ion velocity is mainly controlled by the electric field, which changes little during 0000-0800 UT, the ion speed is almost constant, as shown in Figure 9a. The neutral wind speed increases 50\% from 0000 UT to 0400 UT, and $|\mathbf{V}-\mathbf{U}|$ decreases from 330 to $260 \mathrm{~m} / \mathrm{s}$. Because the direction of the ion velocity is different from the direction of the neutral wind, $|\mathbf{V}-\mathbf{U}|$ changes with $|\mathbf{U}|$ accordingly, but it is not equal to $|\mathbf{V}|-|\mathbf{U}|$. The reason that a secondary peak of the neutral gas heating rate happens at $400 \mathrm{~km}$ altitude is that the heating rate is weighted by the electron density, which maximizes in the $F_{2}$ layer.

[23] The solar wind is the primary driver of geomagnetic events and can strongly affect the magnetosphereionosphere-thermosphere (M-I-T) system, but the M-I-T system is not only a passive receiver. The preconditioning of the system also plays an important role on determining the geoeffectiveness of solar wind drivers. For example, the solar wind conditions are identical at 0100 UT and $0600 \mathrm{UT}$, but the ionosphere-thermosphere (I-T) response is different because the conditions of the I-T system during the previous several hours are different.

\subsection{Effect of Electric Field Temporal Variability}

[24] One of the benefits of models is that they can be run using idealized conditions to quantify input-response functions of the system. This has been done with GITM to better understand the reaction of Joule heating to simple variations in the electric field in a self-consistent thermosphere/ 


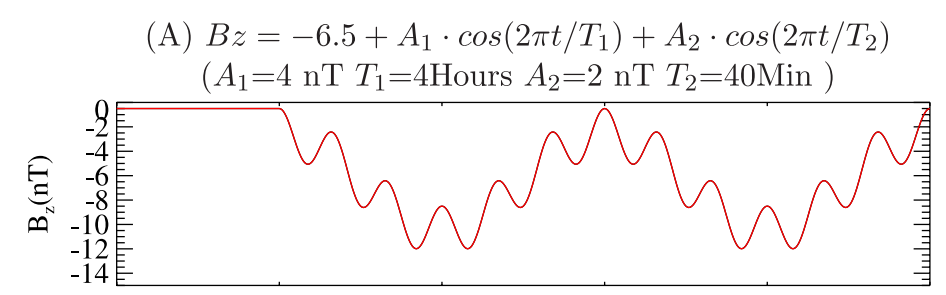

(B) neutral gas heating rate at $400 \mathrm{~km}$ altitude $(\mathrm{K} / \mathrm{s})$

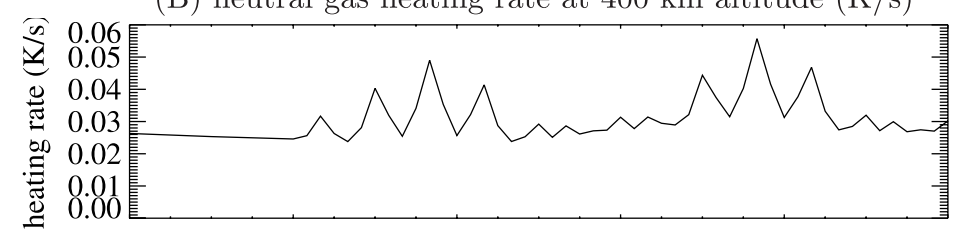

(C) Neutral gas heating rate $(\mathrm{K} / \mathrm{s})$

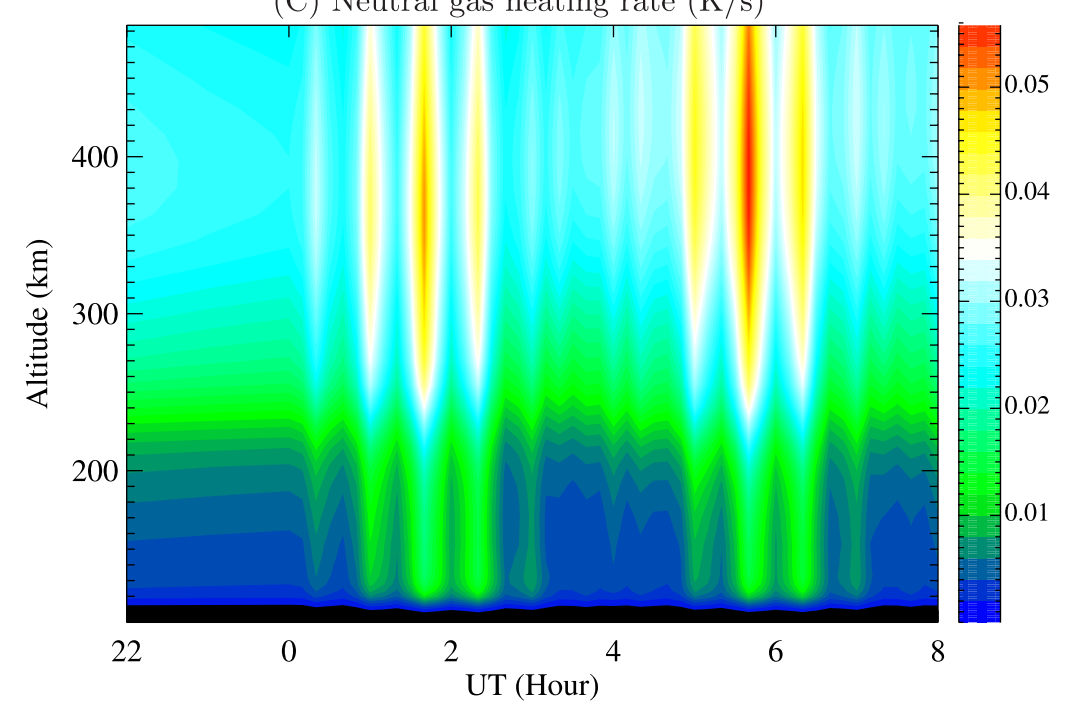

Figure 11. Same as Figure 10, but for the multifrequency case.

ionosphere system. In the previous section, the electric field was changed as a step function and held approximately constant. In this section, the electric field changes such that the average electric field is the same as in the previous section, but it oscillates in time, so there is a quantified variance in the electric field. This is a different methodology than other studies that have investigated the Joule heating during events [e.g., Lu et al., 1995a], or studies that inferred the Joule heating response from only examining the electric field variations [e.g., Codrescu et al., 1995; Crowley and Hackert, 2001; Matsuo et al., 2003].

[25] Three cases have been investigated, in which all input parameters are the same except the high-latitude electric field. As shown in Figure 1, in the first case, $B_{z}$ changes from -0.5 to $-6.5 \mathrm{nT}$ at $0000 \mathrm{UT}$ and then remains constant. In the second case, as shown in Figure 10, a simple sine wave variation with a 4-hour time period (frequency of $0.07 \mathrm{mHz}$ ) and $4 \mathrm{nT}$ amplitude is added to $B_{z}$. Figure 10 shows that the polar average neutral gas heating rate changes instantly with the electric field and the temporal variation has two peaks when $B_{z}$ reaches $-6.5 \mathrm{nT}$. These two peaks at $400 \mathrm{~km}$ altitude are asymmetric, and the second peak is almost $20 \%$ larger than the first one. This is because the variation of the electric field and the variation of the neutral gas heating rate due to the change of $|\mathbf{V}-\mathbf{U}|$, as mentioned in the last section, are in phase. Therefore the resulting neutral gas heating rate is enhanced at the second peak. While this phasing was unplanned, it illustrates that periodic events should be studied closely to determine whether the frequency may be causing stronger (or weaker) thermospheric responses. Thoroughly examining this effect is outside the scope of this study but will be investigated at a later date.

[26] In the third case, as shown in Figure 11, another high-frequency oscillation with a 40-min period (frequency of $0.42 \mathrm{mHz}$ ) and $2 \mathrm{nT}$ amplitude is superposed on the sine wave used in the second case. The neutral gas heating rate changes significantly and the maximum rate is close to $0.056 \mathrm{~K} / \mathrm{s}$, which is $45 \%$ larger than that in the step-change case. The phasing between the 4-hour wave and the neutral gas heating rate due the the variation of $|\mathbf{V}-\mathbf{U}|$ can once again be observed, with the second time period having a larger neutral gas heating rate than the first. When the response to the smaller wavelength is investigated, asymmetries can also be observed. For example, in each of the two 4-hour periods, $B_{z}$ hits $-12 \mathrm{nT}$ twice, separated by $40 \mathrm{~min}$. In each of these cases, the first response is significantly larger than the second. In fact, the second response is approximately the same as when $B_{z}$ is $-8 \mathrm{nT}$ preceding the two $-12 \mathrm{nT}$ peak values. The following $-8 \mathrm{nT}$ peak has very little response. This clearly illustrates that the phasing of the electric field peaks and valleys can 
(A) Average electric field $\bar{E}$ during 00-08 UT $(\mathrm{mV} / \mathrm{m})$

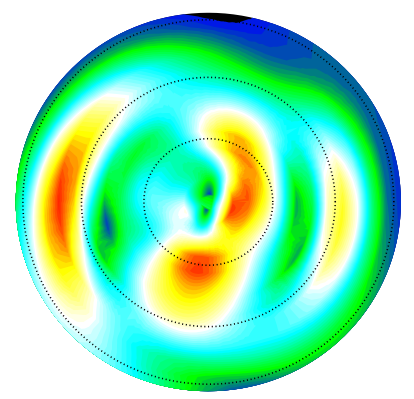

Step change(Max:37.47)

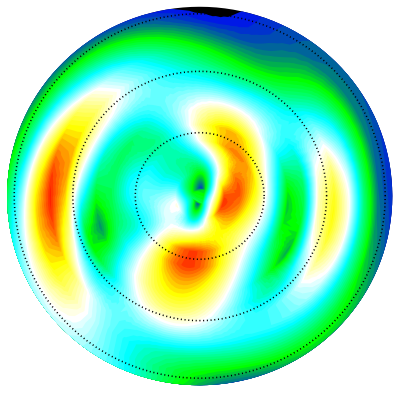

Sine-wave(Max:37.51)

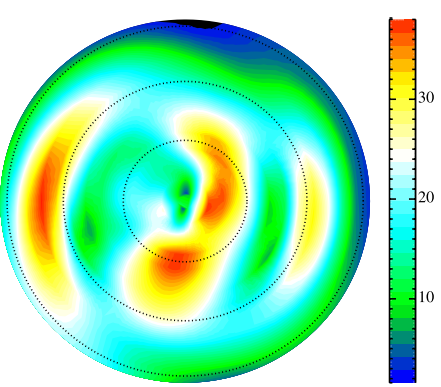

Multi-frequency(Max:37.51)

(B) Standard deviation of electric field $\sigma_{E}$ during 00-08 UT $(\mathrm{mV} / \mathrm{m})$

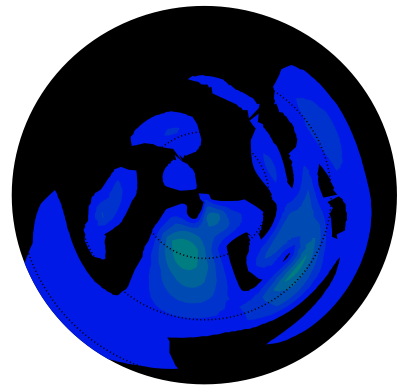

Step change(Max:1.65)

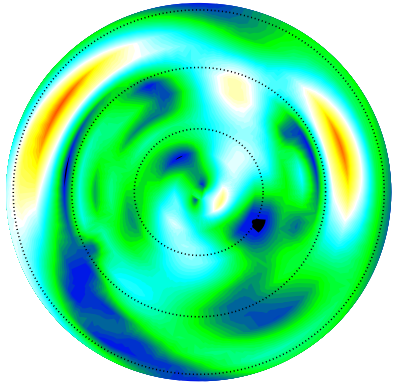

Sine-wave(Max:13.28)

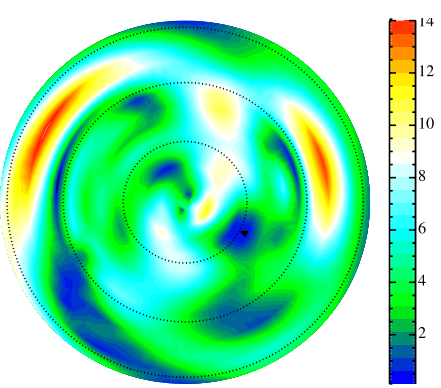

Multi-frequency(Max:14.06)

(C) Average neutral gas heating rate during 00-08 UT (K/s)

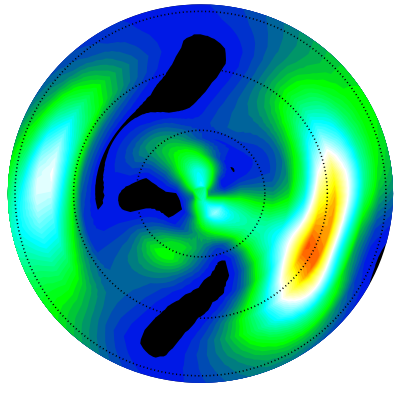

Step change (Max:0.132)

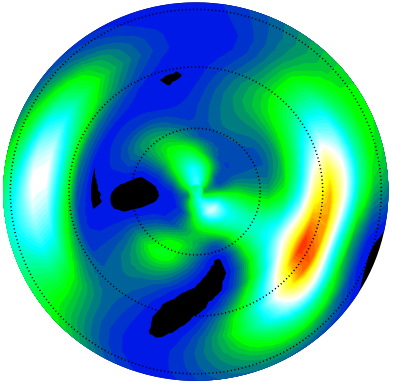

Sine-wave (Max:0.137)

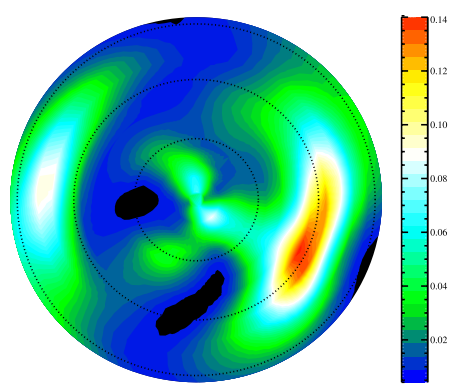

Multi-frequency (Max:0.140)

1

Figure 12. (a) The 8-hour average electric field during 0000-0800 UT; (b) standard deviation of the electric field during 0000-0800 UT; (c) time average neutral gas heating rate during 0000-0800 UT. All of the contours are at $400 \mathrm{~km}$ altitude and the outside rings are $60^{\circ}$ latitude. The maximum value is shown at the bottom of each plot.

have a strong influence of the neutral gas heating rate, so simply estimating Joule heating from the electric field is very difficult.

[27] To determine whether the feedback between the temporal variations of the electric field and Joule heating can be estimated by examining the standard deviation of the electric field (as is done by studies such as Codrescu et al. [1995]), we compare these three cases, which have the same average electric field but different standard deviations. Figure 12 shows the 8-hour averages of the electric field during $0000-0800$ UT for the three cases (top row), which are almost identical. Hence the average electric field does not cause much difference in the neutral gas heating rate between the cases. The standard deviation of the electric field during this period (second row) increases for the sine- wave and multifrequency cases, which causes the neutral gas heating rate (bottom row) to increase. For example, the maximum standard deviation of the electric field rises from 1.65 to $14.06 \mathrm{mV} / \mathrm{m}$, and the maximum 8-hour average neutral gas heating rate correspondingly increases from 0.132 to $0.140 \mathrm{~K} / \mathrm{s}$.

[28] When the spatial distributions of the average electric field $(\bar{E})$ and standard deviation of electric field $\left(\sigma_{E}\right)$ in Figure 12 are examined closely, some differences between them are revealed. For example, in the multifrequency case (third column), $\bar{E}$ has three peaks, specifically at dawn, dusk, and in the polar cap. The $\sigma_{E}$ has a similar distribution, but the dawn and dusk peaks are at lower latitudes than those of $\bar{E}$ and the polar cap peak is not as strong as the dawn and dusk peaks. These characteristics of $\sigma_{E}$ are due to 
(A) Temporal variation of polar average E-field

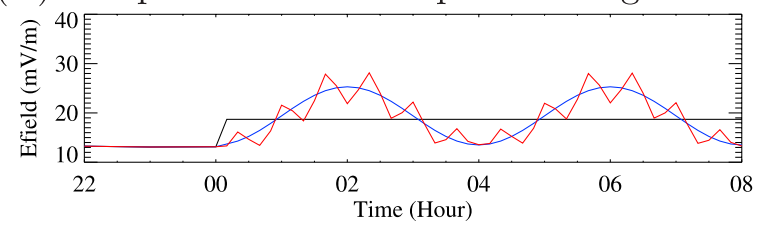

(B) $\sigma_{E} / \bar{E}$

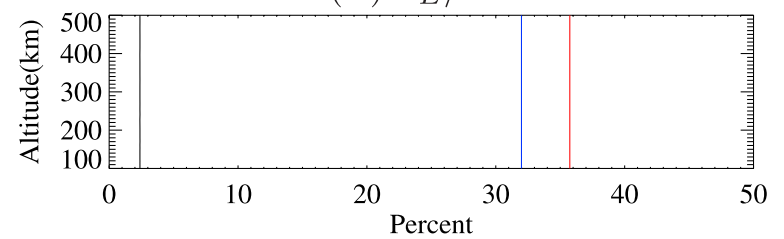

(C) percentage increase of neutral gas heating rate

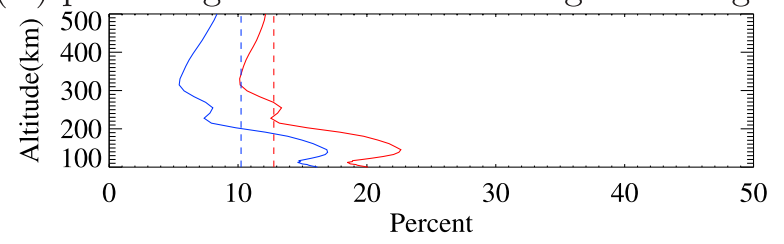

Figure 13. Comparison between the percentage increase of the neutral gas heating rate and $\sigma_{E}^{2} / \bar{E}^{2}$. (a) The temporal variation of the polar average electric field; (b) the ratio of the electric field standard deviation to the average electric field during 0000-0800 UT; (c) the dashed lines show $\sigma_{E}^{2} / \bar{E}^{2}$ and the solid lines show the percentage increase of the neutral gas heating rate compared with the step-change case. The bottom two plots show the polar-averaged values. Black lines are the step-change case; Blue lines are the sinewave case; Red lines are the multifrequency case.

the way the Weimer [1996] model responds to IMF changes. In this study, only $B_{z}$ varies, hence one of the largest changes in the polar cap potential is the expansion and contraction of the polar cap boundary. Owing to the movement of polar cap boundary, in some midlatitude region, such as $60^{\circ}-65^{\circ}$ latitude, there is no potential for weak $B_{z}$ and significant potential for strong $B_{z}$. Therefore the variation of the electric field near the polar cap boundary is larger than that in the center of the polar cap.

[29] In order to quantify the difference among the stepchange, sine-wave, and multifrequency cases, the polar averaged (poleward of $60^{\circ}$ north latitude) electric field standard deviation has been calculated and compared to the average electric field. Figure 13a shows the temporal variation of the polar averaged electric field in the three cases. Figure 13b shows the ratios of the standard deviation of the electric fields to the average electric field. In the simple sine wave case (blue line), the polar average standard deviation is $32 \%$ of the average electric field, and in the multifrequency case (red line), the standard deviation is $36 \%$ of the average electric field. According to the analysis in the work of Codrescu et al. [2000], $Q_{J} \propto \bar{E}^{2}+\sigma_{E}^{2}$. The percentage increase of Joule heating caused by the electric field variation should therefore equal $\sigma_{E}^{2} / \bar{E}^{2}$ if only the $\sigma_{E}$ is important. This is $10 \%$ and $13 \%$ for the sine-wave and multifrequency case, respectively, as shown by the dotted lines in the Figure 13c. However, the calculations show the increase rate in the self-consistent themrosphere/ionosphere system can reach $17 \%$ and $22 \%$ in the sine-wave and multifrequency cases, respectively. Using $\sigma_{E}^{2} / \bar{E}^{2}$ to estimate the percentage increase of Joule heating ignores the neutral wind effect and assumes the electron density distributions are the same for different electric field variation levels. Our results describe the Joule heating variation in a self-consistent thermosphere/ionosphere system. Therefore this discrepancy between $\sigma_{E}^{2} / \bar{E}^{2}$ and the modeled increase in the Joule heating rate, which is highly dependent upon the altitude, is due to the influence of the neutral wind and electron density on Joule heating. The difference between the three cases can also be interpreted in another way: the 4-hour oscillation makes the standard deviation of the electric field increase by $32 \%$ and the neutral gas heating rate increase by $17 \%$. The $40-\mathrm{min}$ time period variation brings only $4 \%$ (36-32\%) additional difference to the standard deviation of the electric field but raises the Joule heating rate by another $5 \%(22-17 \%)$. The comparison between the effect of these two oscillation components shows that the 40-min oscillation is more efficient in changing the neutral gas heating rate than the 4-hour oscillation.

\subsection{Effect of Spatial Resolution}

[30] Since the spatial variability of the electric field is significant [Johnson and Heelis, 2005] and may contribute to Joule heating, the proper spatial resolution within a global model is important in order to capture this smallscale structure. Using the Weimer [1996] model as the highlatitude electric field driver, GITM has been run under three commonly used resolutions $\left(5^{\circ} \times 5^{\circ}, 5^{\circ} \times 2.5^{\circ}, 5^{\circ} \times 1.25^{\circ}\right.$, longitude $\times$ latitude). The TIEGCM runs at $5^{\circ} \times 5^{\circ}$, so this is the baseline case, to determine how much Joule heating is missed in typical model runs. The three cases (step-change, sine-wave, and multifrequency cases) are run to determine the net effect of temporal variability and spatial resolution. Figure 14 shows the spatial distribution of the 8-hour (0000-0800 UT) averaged neutral gas heating rate at $400 \mathrm{~km}$ altitude in the multifrequency case. It is notable that the maximum neutral gas heating rate increases with the resolution from 0.110 to $0.150 \mathrm{~K} / \mathrm{s}$. The higher resolution catches smaller structures and gives a more precise specification of the maximum values in the electric field and particle precipitation. When the resolution is not high enough, the maximum values can be lost in the large spaces between the grid points and therefore the low-resolution simulation underestimates the maximum neutral gas heating rate. Indeed, not only has the magnitude changed with resolution, but the spatial distribution has some small differences. For example, the dawnside maximum shifts to the nightside when the resolution increases. More subgrid heating at earlier local times is a reasonable explanation for this result. This is not necessarily a general conclusion, though, since the location of the maximum heating rate is quite dependent on the specific driving conditions. The significance of this result is that low resolution simulations not only underestimate the magnitude of the neutral gas heating rate but also may give imprecise information about the spatial distribution.

[31] Figure 15 shows the altitude profile of the polaraveraged value of the 8-hour average neutral gas heating rate and the percentage increase when compared with the lowest resolution $\left(5^{\circ} \times 5^{\circ}\right)$. In each case, the neutral gas 


\section{Neutral gas heating rate with different spatial resolution $(\mathrm{K} / \mathrm{s})$}
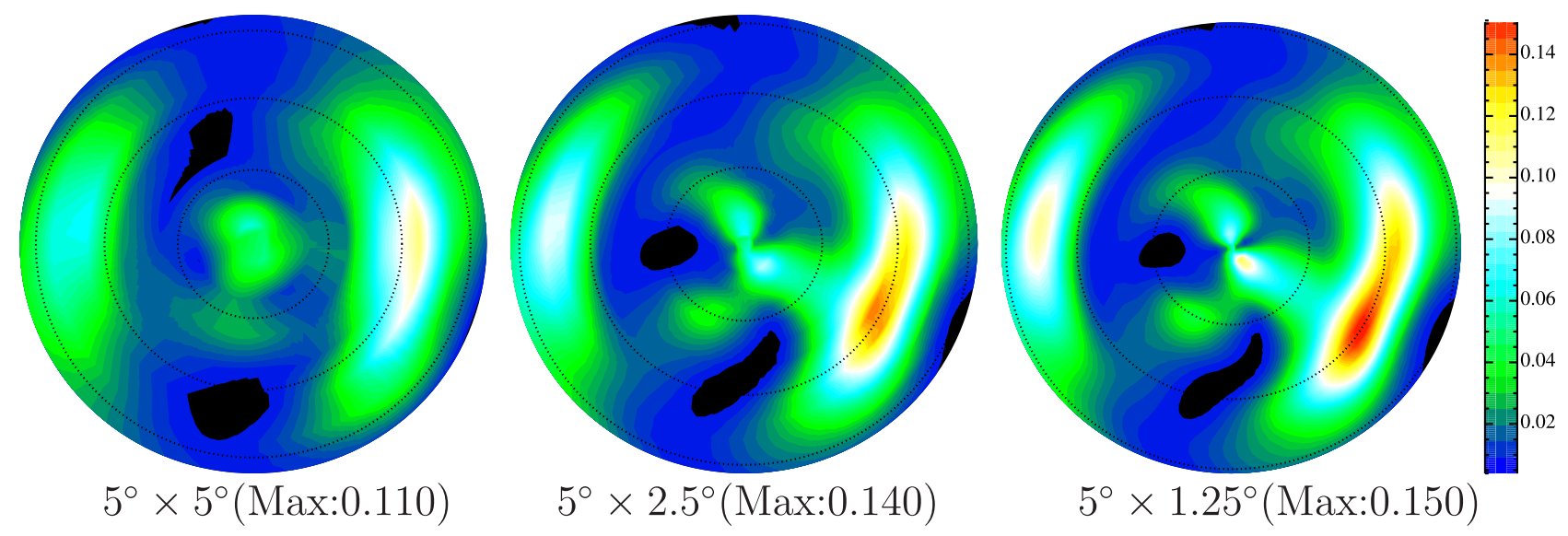

Figure 14. Eight-hour average (0000-0800 UT) neutral gas heating rate $(\mathrm{K} / \mathrm{s})$ at $400 \mathrm{~km}$ altitude with three different resolutions in the multifrequency case. The left figure has resolution of $5^{\circ}$ longitude by $5^{\circ}$ latitude, the middle figure is $5^{\circ}$ longitude by $2.5^{\circ}$ latitude, and the right figure is $5^{\circ}$ longitude by $1.25^{\circ}$ latitude.

heating rate increases with resolution. For example, at $200 \mathrm{~km}$, it can cause approximately a $20 \%$ difference. The percentage increase from $5^{\circ} \times 5^{\circ}$ to $5^{\circ} \times 2.5^{\circ}$ is larger than that from $5^{\circ} \times 2.5^{\circ}$ to $5^{\circ} \times 1.25^{\circ}$, which indicates that the solution is converging (but is most likely not converged). Although this may not be the true case, since the electrodynamics models that are available today [e.g., Weimer, 1996; Fuller-Rowell and Evans, 1987] have an approximately $1^{\circ}$ latitudinal resolution and are smeared out because they are averaged patterns. The right column of Figure 15 shows the percentage increase of the neutral gas heating rate when compared with the results using the lowest resolution $5^{\circ} \times 5^{\circ}$. In the multifrequency case (C) at $400 \mathrm{~km}$ altitude, with $2.5^{\circ}$ latitudinal resolution, there is an approximate $12 \%$ increase in the neutral gas heating rate (compared to $5^{\circ}$ latitudinal resolution), while with $1.25^{\circ}$ resolution, the increase is about $20 \%$. This means that structures with sizes between $1.25^{\circ}$ and $2.5^{\circ}$ latitude make an $8 \%$ difference to the neutral gas heating rate, which is comparable to the effect of structures between $2.5^{\circ}$ and $5.0^{\circ}(12 \%)$. More precise knowledge about the scale dependence needs a spectral analysis of the Joule heating spatial distribution using high-resolution electric field and particle precipitation drivers, which will be a part of our future work. To truly capture all of the small-scale effects, both the electrodynamical model and GCM would have to have extremely high resolution, since auroral arcs are known to be as small as meters across. Capturing these features in a global model is quite impossible at this time, due to a lack of global observations and a lack of computational resources. Therefore GCMs will continue to not be grid-converged, as evidenced above.

[32] When comparing the altitude profile of the neutral gas heating rate under low resolution in the step case with that under the high resolution in the multifrequency case, as shown in Figure 16, the difference is close to $50 \%$ around $180 \mathrm{~km}$ altitude. This increased ratio is due to the combined effects of temporal variation and spatial resolution. It is interesting to note that Joule heating within TIEGCM and CTIP is multiplied by approximately 1.5 for the summer hemisphere and 2.5 for the winter hemisphere, respectively [Emery et al., 1999], to increase it to more observed levels, based on the work by Codrescu et al. [1995] and Codrescu et al. [2000]. This is similar to the factor that is arrived here by running in higher resolution and capturing the temporal variability of the electric field. One should be careful comparing these results to Emery et al. [1999], since we describe the ratio of the neutral gas heating rate and Emery et al. [1999] uses the ratio of the Joule heating energy deposition rate. As was discussed in section 3.1.2, it is the neutral density that makes the neutral gas heating rate and the Joule heating energy deposition rate different. Since neutral density is too heavy to follow the high-frequency variation of ions and also does not have as many small-scale structures as the electric field, the neutral density varies little with the $E$ field temporal variation and spatial resolution. As shown in Figure 16, the percentage difference of mass density between these cases is relatively small and the calculated percentage increase of the energy deposition rate is very close to the percentage increase of the neutral gas heating rate. Therefore the comparison between our percentage increase of the neutral gas heating rate and the percentage increases of the energy deposition rate in the work of Emery et al. [1999] is appropriate.

\subsection{Effect of Vertical Velocity Difference}

[33] In the polar region, the magnetic field is almost vertical and the $\mathbf{E} \times \mathbf{B}$ drift is typically considered to be horizontal. Therefore in the NCAR GCMs [e.g., Roble et al., 1982] the Joule heating is calculated in the horizontal plane:

$$
Q_{J H}=\lambda_{X X}\left(V_{e}-U_{e}\right)^{2}+\lambda_{Y Y}\left(V_{n}-U_{n}\right)^{2}
$$

where $Q_{J H}$ is the Joule heating energy deposition rate, $\lambda_{X X}$ and $\lambda_{Y Y}$ are ion drag coefficients. $V_{e}$ and $U_{e}$ are the zonal ion and neutral wind velocities, respectively. $V_{n}$ and $U_{n}$ are the corresponding meridional winds. Nevertheless, Deng and Ridley [2006b] showed that the ion drifts can have a 
(A) Step-change case
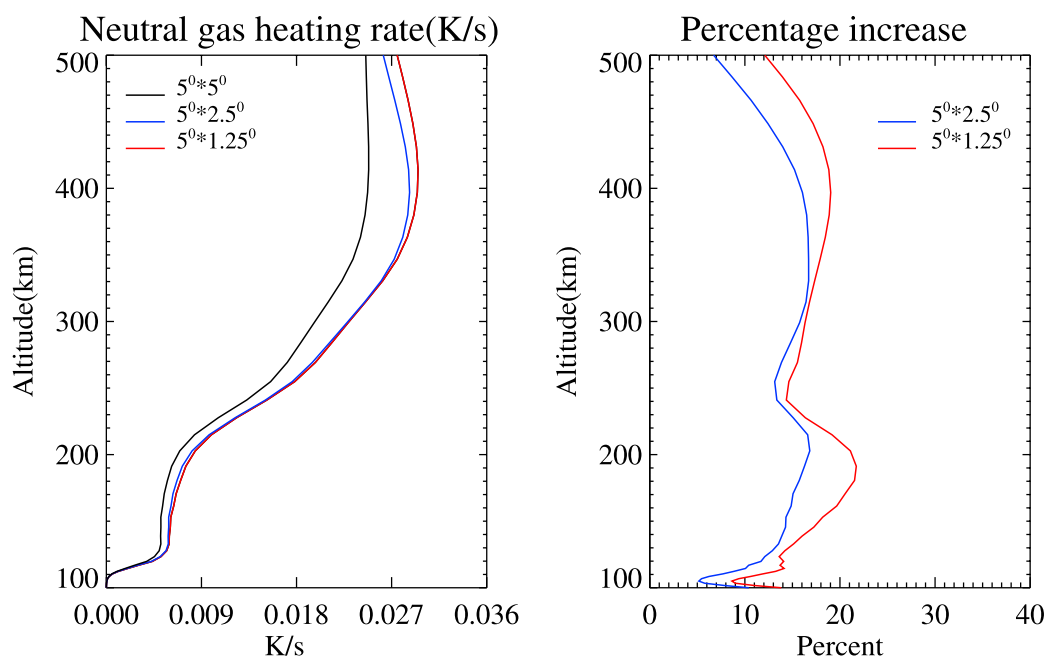

(B) Sine-wave case
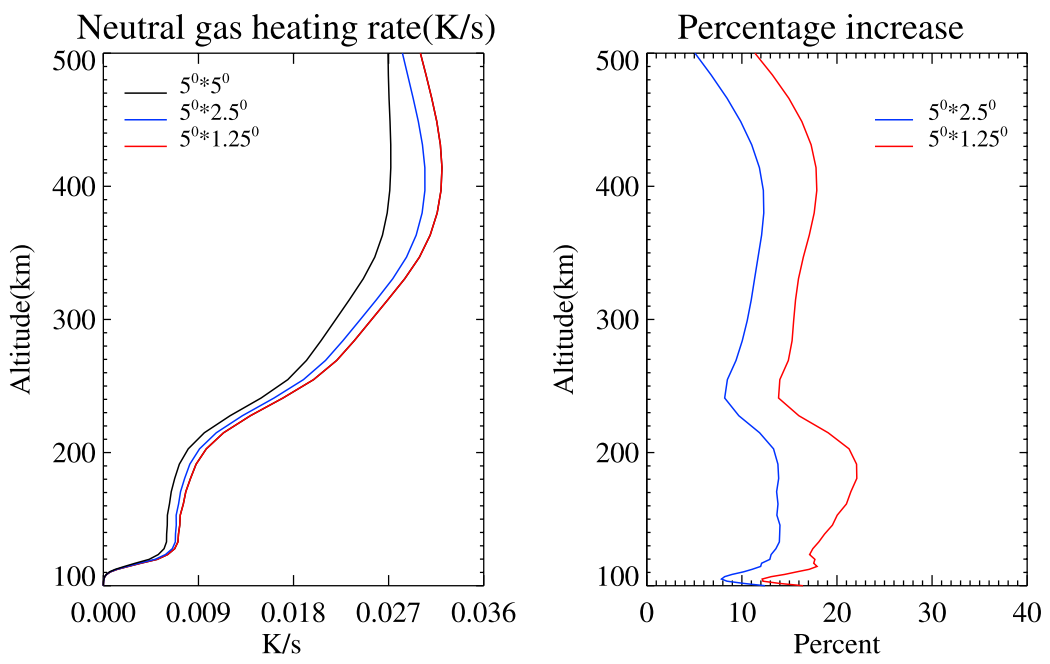

(C) Multi-frequency case
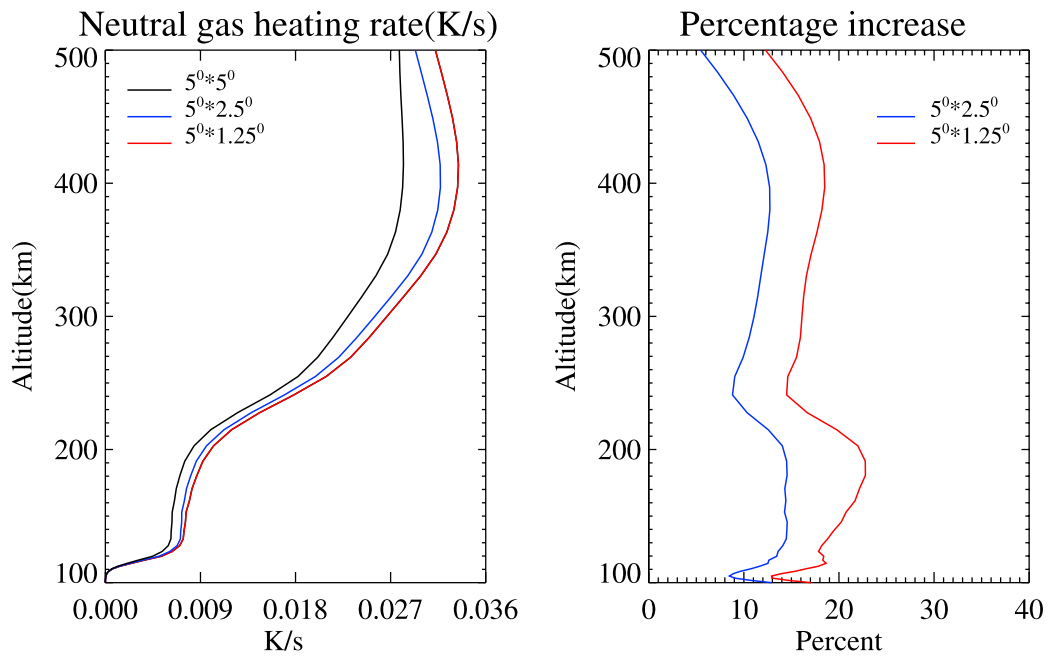

Figure 15. (left) Altitude profile of the polar average of 8-hour average neutral gas heating rate with three different resolutions; (right) percentage increase when compared with the lowest resolution $\left(5^{\circ} \times 5^{\circ}\right)$ in each case. Top row is the step-change case, middle row is the sine-wave case, and the bottom row is the multifrequency case. The black lines represent the resolution of $5^{\circ}$ longitude by $5^{\circ}$ latitude, the blue lines represent $5^{\circ}$ longitude by $2.5^{\circ}$ latitude and the red lines represent $5^{\circ}$ longitude by $1.25^{\circ}$ latitude. 

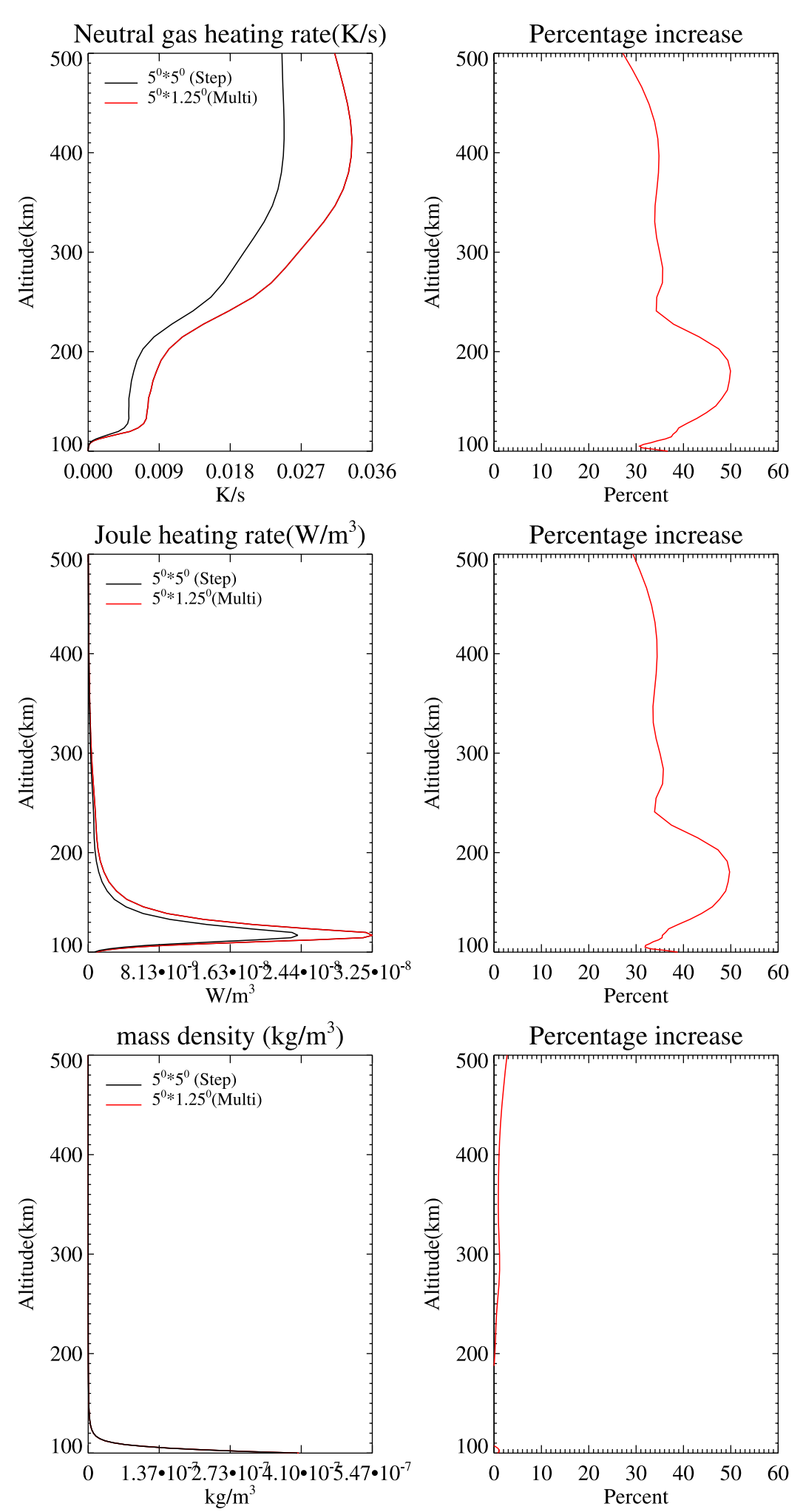

Figure 16. Comparison between the multifrequency case under high resolution and the step-change case under low resolution. (left) Altitude profile of the polar average of 8-hour average neutral gas heating rate; (right) percentage increase when compared with the lowest resolution $\left(5^{\circ} \times 5^{\circ}\right)$ in stepchange case. Top row is the neutral gas heating rate, middle row is the Joule heating energy deposition rate, and the bottom row is the mass density. 
neutral gas heating rate in $2-\mathrm{D}$ (left) and $3-\mathrm{D}$ (Right) $(\mathrm{K} / \mathrm{s})$
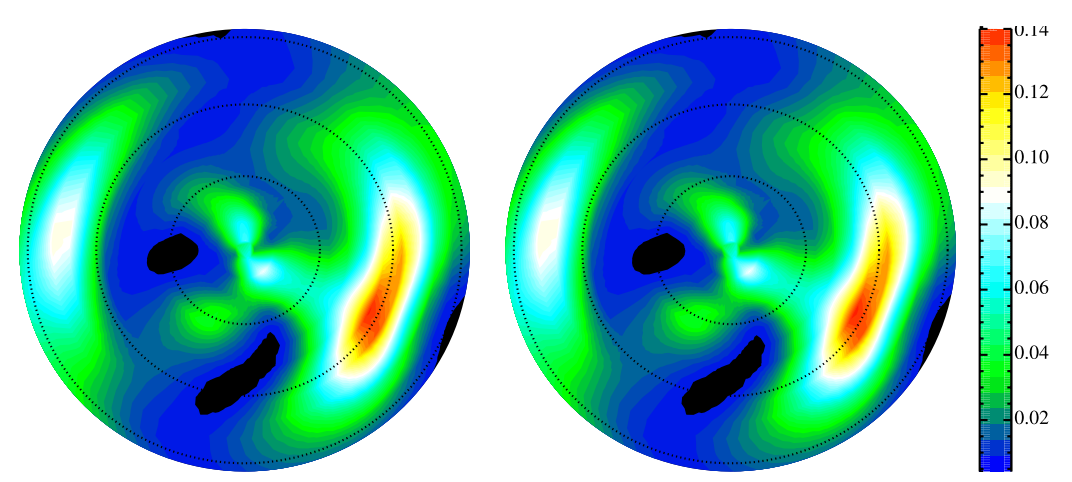

\section{Difference $(\mathrm{K} / \mathrm{s})$}

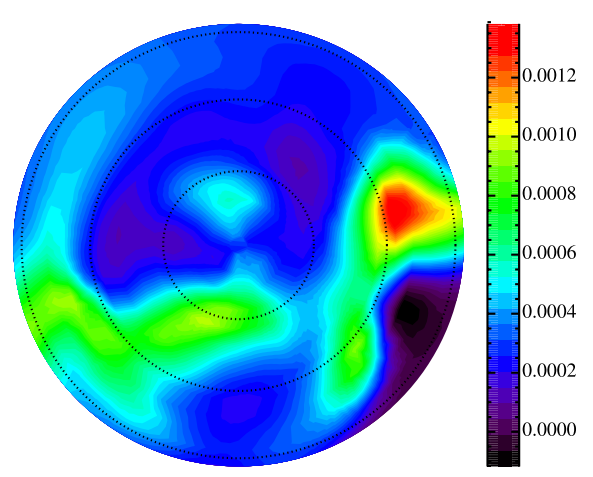

Figure 17. (left) Eight-hour average (0000-0800 UT) neutral gas heating rate at $400 \mathrm{~km}$ altitude when calculated in the two-dimensional (2-D) and 3-D manners. (right) The difference between 2-D and 3-D results is shown on the right.

significant vertical component considering the change of the geomagnetic dip angle with latitude and the significant convection electric field at auroral latitudes. In order to investigate the importance of the vertical velocity difference between ion and neutral flows on Joule heating, we calculate the neutral gas heating rate in two ways: one with the contribution of vertical velocities (3-D manner), as described in equation (1), and one without (2-D manner). As shown in Figure 17, the spatial distributions of the neutral gas heating rate calculated in 2-D and 3-D manners are very similar, and the difference between them is more than one order of magnitude smaller than the neutral gas heating rate and is not evenly distributed.

[34] Figure 18a shows that the polar average percentage increase of 3-D results compared with the 2-D results is close to $2 \%$ at $400 \mathrm{~km}$. This is much smaller than the other two factors (temporal variability and spatial resolution) and seems to be negligible. Interestingly, observed vertical neutral winds sometimes can be significant [Wescott et al., 2006; Oyama et al., 2005] and the contribution of the vertical velocity difference can be larger than our simulation. The altitude profile of the percentage increase minimizes in the range of $100-200 \mathrm{~km}$ altitudes, which is also different from the other two factors. This is because the vertical ion convection is caused mainly by the vertical component of the $\mathbf{E} \times \mathbf{B}$ drift. Above $200 \mathrm{~km}$, the ion convection is mainly controlled by the $\mathbf{E} \times \mathbf{B}$ drift. But at lower altitudes, other factors, such as neutral drag, are also important and make the ion convection depart from the $\mathbf{E} \times \mathbf{B}$ drift [Richmond et al., 2003]. Therefore the low-altitude vertical ion convection is not as significant as it is at high altitudes.

[35] Figure 18b shows the averaged value of the neutral gas heating rate and the percentage increase at the middle and low latitudes $\left(-60^{\circ}<\right.$ Lat $\left.<60^{\circ}\right)$. While the neutral gas heating rate is more than four times smaller than the polar average, the percentage increase caused by the vertical velocity can reach $15 \%$, which is larger than the polar average. This result indicates that, while the magnitude of the neutral gas heating rate at middle and low latitudes is smaller than that at high latitudes, the relative importance of the vertical velocity difference is larger. This result may be straightforward, but it shows that it may be important to include the vertical component of the Joule heating calculation at middle and low latitudes, especially during time periods with strong vertical drifts, such as storm times.

\section{Discussion and Conclusion}

[36] The above results describe the effects of electric field changes on the thermospheric Joule heating in the selfconsistent thermosphere/ionosphere system. They show how the temporal variability, spatial resolution, and model assumptions may affect the Joule heating. These results are summarized and discussed here.

[37] Most studies of Joule heating have examined the Joule heating energy deposition rate and not the time rate of change of the thermospheric temperature due to Joule heating. The neutral gas heating rate is proportional to the ratio of the Joule heating energy deposition rate and the neutral density. Since the neutral density exponentially decreases with altitude, the neutral gas heating rate and the Joule heating energy deposition rate have totally different altitude profiles. While the maximum Joule heating energy deposition rate happens at $120 \mathrm{~km}$ altitude (i.e., most of the energy is deposited at $120 \mathrm{~km}$ altitude), the neutral gas heating rate maximizes around $400 \mathrm{~km}$ altitude (i.e., the temperature is changing fastest due to Joule heating at $400 \mathrm{~km}$ ). Since the main reason to examine Joule heating is to examine how the temperature structure (and therefore the density structure) changes, this study focuses on the neutral gas heating rate and not the energy deposition rate.

[38] When the IMF $B_{z}$ increases from -0.5 to $-6.5 \mathrm{nT}$, the polar-averaged Joule heating energy deposition rate increases by $70 \%$ abruptly. While Joule heating is strongly affected by the convection electric field, both the neutral wind and the electron density significantly alter the spatial distribution of Joule heating, and consequently make it different from the spatial distribution of the electric field. As a result of the neutral wind ramp-up, there is temporal variation of the Joule heating energy deposition rate when the electric field is mostly constant. 

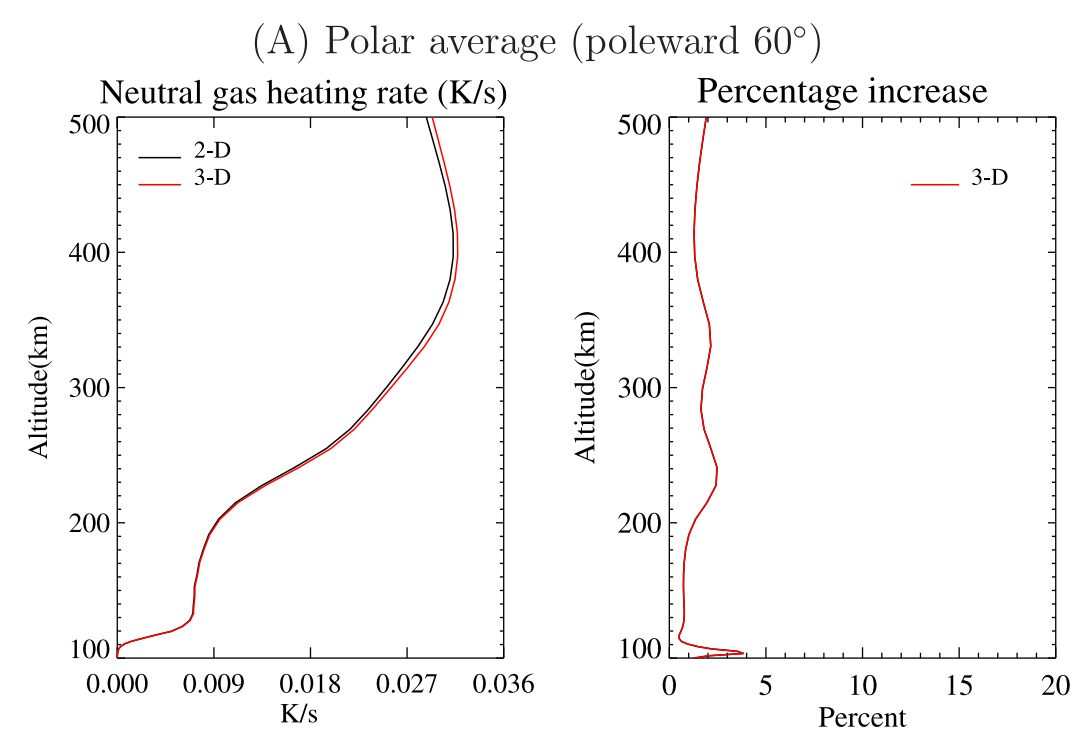

(B) Mid- an low- latitude average $\left(-60^{\circ}<\right.$ Lat $\left.<60^{\circ}\right)$
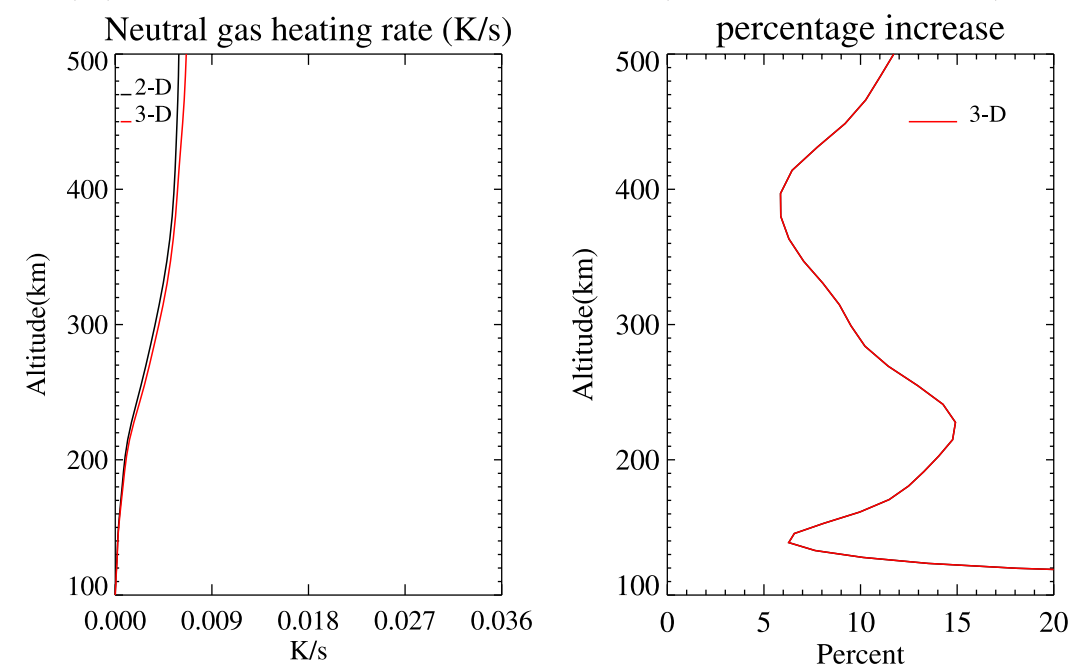

Figure 18. (a) Altitude profile of polar average of the 8-hour average neutral gas heating rate calculated in 2-D and 3-D manners (left) and percentage increase of 3-D results compared with the 2-D results (right). (b) The same as Figure 18a except they are middle- and low-latitude averages $\left(-60^{\circ}<\right.$ Lat $\left.<60^{\circ}\right)$. The black lines represent the 2-D results and the red lines represent the 3-D results.

[39] The neutral gas heating rates are compared when running with three time series of electric fields, which have the same temporal average but different standard deviations. In both the sine-wave and the multifrequency cases, there is a strong asymmetry of the neutral gas heating rates between the two large southward IMF $B_{z}$ time periods. The second period results in an almost $20 \%$ larger neutral gas heating rate than the first period because the 4-hour time period electric field variation is in phase with the variation of the neutral wind changes. This shows that the preconditioning of the thermospheric state may significantly alter Joule heating. The neutral gas heating rate increases with the electric field temporal variability, while the percentage increase depends on both the magnitude and frequency of the electric field variation. As discussed in section 3.2, owing to the effect of the neutral winds and electron density, the percentage increase related to the temporal variability is not exactly equal to $\sigma_{E}^{2} / \bar{E}^{2}$, which is normally used to describe the effect of electric field temporal variability on Joule heating [e.g., Codrescu et al., 1995; Crowley and Hackert, 2001; Matsuo et al., 2003], and is strongly dependent upon altitude, maximizing around $150 \mathrm{~km}$. Above approximately $200 \mathrm{~km}, \sigma_{E}^{2} / \bar{E}^{2}$ is a overestimation of the heating change rate, while, below this altitude, it may underestimate the heating rate by almost a factor of two.

[40] In this study, some simple sine-wave oscillations have been added to represent the different variation levels of the electric field, which are obviously different from real cases. In reality, the oscillations include many different frequencies and amplitude components and can often be close to random noise. Using AMIE as the high-latitude driver may give a more realistic estimation of the impact of the temporal electric field variability on Joule heating. However, in AMIE, it is hard to separate the effect of 
electric field temporal variability with variations in the particle precipitation. Therefore special caution should be taken to choose the right time period when investigating the effects of electric temporal variability on Joule heating using AMIE.

[41] Comparison between three model resolutions shows that the maximum neutral gas heating rate increases when the resolution increases. At $200 \mathrm{~km}$ altitude, the polar averaged neutral gas heating rate increases by $20 \%$ when the latitudinal resolution increases from $5^{\circ}$ to $1.25^{\circ}$. As shown in section 3.3 , the polar average neutral gas heating rate with the highest latitudinal resolution $\left(1.25^{\circ}\right)$ and highest temporal variability (multifrequency case) is $50 \%$ larger than that with the lowest latitudinal resolution $\left(5^{\circ}\right)$ and the lowest temporal variability (step-change case). The altitude profile of the percentage increase shows both temporal variability and spatial resolution can strongly affect the neutral gas heating rate around $200 \mathrm{~km}$ altitude.

[42] Even though our results show that increasing the spatial resolution of the GCMs can increase the neutral gas heating rate, this result is limited by the resolution and type of the electrodynamic models acting as the high-latitude drivers to GCMs. For our studies, the high-latitude electric field driver is from the Weimer [1996] model and the particle precipitation model is from Fuller-Rowell and Evans [1987]. They are averaged and smoothed empirical models showing some limited variability. Styers et al. [2004] and Lummerzheim et al. [2004] showed, from both observational and modeling points of view, that the smallscale aurora can contribute to Joule heating significantly. Small-scale aurora is not present in large scale averages and the empirical precipitation models underestimate the spatial variability of the precipitation. Therefore our estimate of $20 \%$ of the Joule heating, being missed by having a $5^{\circ}$ latitudinal resolution, is an underestimation of the effect of spatial resolution on the calculated Joule heating due to the inherent limitation of spatially averaged electrodynamic models.

[43] The contribution of the vertical velocity difference is smaller than that of the temporal variation and the spatial resolution, and appears to be negligible at high latitudes. The altitude profile of the percentage increase of the neutral gas heating rate caused by the vertical velocity difference is also different from the other two factors and maximizes between $300 \mathrm{~km}$ and $400 \mathrm{~km}$ altitude. While the middleand low-latitude average of the neutral gas heating rate is smaller than the polar average value $(0.008 \mathrm{~K} / \mathrm{s}$ versus $0.033 \mathrm{~K} / \mathrm{s}$ ), the percentage increase of the middle- and low-latitude average value due to the vertical velocity difference is larger than that of the polar average value (15\% versus $3 \%)$. This result indicates that the vertical velocity difference contribution to the neutral gas heating rate is relatively more important at middle and low latitudes than at high latitudes.

[44] It is very challenging to precisely simulate the thermosphere/ionosphere response to the energy input from the magnetosphere. One primary limitation comes from electrodynamic models, which are the high-latitude drivers for GCMs. In the future, if the subgrid structures of both electric field and particle precipitation can be parameterized appropriately in the driver models, it is potentially possible for GITM to simulate the small-scale structures in the thermosphere/ionosphere and help to understand the relative importance of different mechanisms causing complex disturbances of the thermosphere [Lühr et al., 2004].

\section{Appendix A}

[45] While the GITM code was described in detail by Ridley et al. [2006], the main equations are again repeated here for completeness. The variables in the model are

$\mathrm{t}$ time

$\theta$ north latitude;

$\phi \quad$ east longitude;

$\mathrm{r}$ radial distance measured from the center of the Earth;

$N_{e}$ electron density;

$N_{s}$ number density of species s;

$\mathcal{N}_{s} \ln \left(N_{s}\right)$

$\rho$ neutral mass density;

$\rho_{i}$ ion mass density;

$\mathbf{v}$ ion velocity;

$v_{r}$ radial component of the ion velocity;

u neutral velocity;

$u_{\theta}$ northward neutral velocity;

$u_{\phi}$ eastward neutral velocity;

$u_{r}$ radial neutral velocity;

$u_{r, s}$ radial neutral velocity of species s;

$T$ temperature;

$\mathcal{T} p / \rho$;

$\Omega$ angular velocity of the planet;

$M_{s}$ molecular mass of species s;

$g$ acceleration of gravity;

$k$ Boltzmann constant;

$\nu_{\text {in }}$ ion-neutral collision frequency;

$D_{q s}$ diffusion coefficient;

$\eta$ coefficient of viscosity;

E externally generated electric field

(i.e., magnetospheric);

B magnetic field;

$B$ magnitude of $|\mathbf{B}|$;

b direction of the magnetic field;

$P_{i}$ ion pressure;

$P_{e}$ electron pressure;

$e$ electron charge;

$K_{e}$ eddy diffusion coefficient.

\section{A1. Continuity Equation}

[46] For each species the vertical continuity equation in spherical coordinates is

$$
\frac{\partial \mathcal{N}_{s}^{V}}{\partial t}=-\frac{\partial u_{r, s}}{\partial r}-\frac{2 u_{r, s}}{r}-u_{r, s} \frac{\partial \mathcal{N}_{s}}{\partial r}
$$

[47] The horizontal continuity equation is

$$
\begin{aligned}
\frac{\partial N_{s}^{H}}{\partial t}= & -N_{s}\left(\frac{1}{r} \frac{\partial u_{\theta}}{\partial \theta}+\frac{1}{r \cos \theta} \frac{\partial u_{\phi}}{\partial \phi}-\frac{u_{\theta} \tan \theta}{r}\right) \\
& -\frac{u_{\theta}}{r} \frac{\partial N_{s}}{\partial \theta}-\frac{u_{\phi}}{r \cos \theta} \frac{\partial N_{s}}{\partial \phi}
\end{aligned}
$$


[48] The source term $\frac{\partial N_{s}^{S}}{\partial t}$ for the neutral density of species $s$ includes the eddy diffusion and chemical sources and losses:

$$
\frac{\partial N_{s}^{S}}{\partial t}=\frac{\partial}{\partial r}\left[N_{s} K_{e}\left(\frac{\partial \mathcal{N}_{s}}{\partial r}-\frac{\partial \mathcal{N}}{\partial r}\right)\right]+C_{S}
$$

[49] The total change rate of density is

$$
\frac{\partial N_{s}}{\partial t}=N_{s} \frac{\partial \mathcal{N}_{s}^{V}}{\partial t}+\frac{\partial N_{s}^{H}}{\partial t}+\frac{\partial N_{s}^{S}}{\partial t}
$$

\section{A2. Momentum Equations}

[50] In rotating spherical coordinates the vertical momentum equation for each species is

$$
\begin{gathered}
\frac{\partial u_{r, s}}{\partial t}+u_{r, s} \frac{\partial u_{r, s}}{\partial r}+\frac{u_{\theta}}{r} \frac{\partial u_{r, s}}{\partial \theta}+\frac{u_{\phi}}{r \cos (\theta)} \frac{\partial u_{r, s}}{\partial \phi}+\frac{k}{M_{s}} \frac{\partial T}{\partial r}+T \frac{k}{M_{s}} \frac{\partial \mathcal{N}_{s}}{\partial r} \\
=g+\mathcal{F}_{s}+\frac{u_{\theta}^{2}+u_{\phi}^{2}}{r}+\cos ^{2}(\theta) \Omega^{2} r+2 \cos (\theta) \Omega u_{\phi},
\end{gathered}
$$

$\mathcal{F}_{S}$ contains the forces due to the ion-neutral [Rees, 1989] and the neutral-neutral friction in the vertical direction [Colegrove et al., 1966]:

$$
\mathcal{F}_{s}=\frac{\rho_{i}}{\rho_{s}} \nu_{\text {in }}\left(v_{r}-u_{r, s}\right)+\frac{k T}{M_{s}} \sum_{q \neq s} \frac{N_{q}}{N D_{q s}}\left(u_{r, q}-u_{r, s}\right)
$$

[51] The eastward momentum equation is

$$
\begin{gathered}
\frac{\partial u_{\phi}}{\partial t}+u_{r} \frac{\partial u_{\phi}}{\partial r}+\frac{u_{\theta}}{r} \frac{\partial u_{\phi}}{\partial \theta}+\frac{u_{\phi}}{r \cos \theta} \frac{\partial u_{\phi}}{\partial \phi}+\frac{1}{r \cos \theta} \frac{\partial \mathcal{T}}{\partial \phi}+\frac{\mathcal{T}}{\rho r \cos \theta} \frac{\partial \rho}{\partial \phi} \\
=\frac{\mathcal{F}_{\phi}}{\rho}+\frac{u_{\phi} u_{\theta} \tan \theta}{r}-\frac{u_{r} u_{\phi}}{r}+2 \Omega u_{\theta} \sin \theta-2 \Omega u_{r} \cos \theta, \quad(\mathrm{A} 7)
\end{gathered}
$$

[52] The northward momentum equation is

$$
\begin{aligned}
\frac{\partial u_{\theta}}{\partial t} & +u_{r} \frac{\partial u_{\theta}}{\partial r}+\frac{u_{\theta}}{r} \frac{\partial u_{\theta}}{\partial \theta}+\frac{u_{\phi}}{r \cos \theta} \frac{\partial u_{\theta}}{\partial \phi}+\frac{1}{r} \frac{\partial \mathcal{T}}{\partial \theta}+\frac{\mathcal{T}}{\rho r} \frac{\partial \rho}{\partial \theta} \\
= & \frac{\mathcal{F}_{\theta}}{\rho}+\frac{u_{\phi}^{2} \tan \theta}{r}-\frac{u_{\theta} u_{r}}{r}-\Omega^{2} r \cos \theta \sin \theta-2 \Omega u_{\phi} \sin \theta .
\end{aligned}
$$

The force terms due to ion-neutral friction and viscosity are

$$
\begin{aligned}
& \mathcal{F}_{\theta}=\rho_{i} \nu_{i n}\left(v_{\theta}-u_{\theta}\right)+\frac{\partial}{\partial r} \eta \frac{\partial u_{\theta}}{\partial r} \\
& \mathcal{F}_{\phi}=\rho_{i} \nu_{i n}\left(v_{\phi}-u_{\phi}\right)+\frac{\partial}{\partial r} \eta \frac{\partial u_{\phi}}{\partial r}
\end{aligned}
$$

\section{A3. Energy Equation}

[53] The vertical thermodynamic equation is

$$
\frac{\partial \mathcal{T}^{V}}{\partial t}=-u_{r} \frac{\partial \mathcal{T}}{\partial r}-(\gamma-1) \mathcal{T}\left(\frac{2 u_{r}}{r}+\frac{\partial u_{r}}{\partial r}\right)
$$

[54] The horizontal thermodynamic equation is

$$
\begin{aligned}
\frac{\partial \mathcal{T}^{H}}{\partial t}= & -\frac{u_{\phi}}{r \cos \theta} \frac{\partial \mathcal{T}}{\partial \phi}-\frac{u_{\theta}}{r} \frac{\partial \mathcal{T}}{\partial \theta} \\
& -(\gamma-1) \mathcal{T}\left(\frac{1}{r} \frac{\partial u_{\theta}}{\partial \theta}+\frac{1}{r \cos \theta} \frac{\partial u_{\phi}}{\partial \phi}-\frac{u_{\theta} \tan \theta}{r}\right)
\end{aligned}
$$

[55] The thermal energy source term is

$$
\begin{aligned}
\frac{\partial \mathcal{T}^{S}}{\partial t}= & \frac{k}{c_{v} \rho}\left(Q_{E U V}+Q_{N O}+Q_{O}\right. \\
& \left.+\frac{\partial}{\partial r}\left(\left(\kappa_{c}+\kappa_{e d d y}\right) \frac{\partial T}{\partial r}\right)+N_{e} \bar{m}_{i} \nu_{i n}(\mathbf{v}-\mathbf{u})^{2}\right)
\end{aligned}
$$

[56] The total temperature change rate is

$$
\frac{\partial \mathcal{T}}{\partial t}=\frac{\partial \mathcal{T}^{V}}{\partial t}+\frac{\partial \mathcal{T}^{H}}{\partial t}+\frac{\partial \mathcal{T}^{S}}{\partial t}
$$

\section{A4. Equations for Ions}

[57] The ion momentum equation can be solved for the ion velocity:

$$
\begin{aligned}
\mathbf{v}= & \mathbf{u} \cdot \mathbf{b}+\frac{1}{\nu_{i n}}\left(\mathbf{g} \cdot \mathbf{b}-\frac{\nabla\left(P_{i}+P_{e}\right) \cdot \mathbf{b}}{\rho_{i}}\right) \\
& +\frac{\rho_{i} \nu_{i n} \mathbf{A}+e N_{e} \mathbf{A} \times \mathbf{B}}{\rho_{i}^{2} \nu_{i n}^{2}+e^{2} N_{e}^{2} B^{2}}
\end{aligned}
$$

where

$$
\mathbf{A}=\rho_{i} \mathbf{g}_{\perp}+e N_{e} \mathbf{E}_{\perp}-\nabla\left(P_{i}+P_{e}\right)_{\perp}+\rho_{i} \nu_{i n} \mathbf{u}_{\perp}
$$

[58] The electron energy equation is the same as in the work of Schunk and Nagy [1978].

[59] Acknowledgments. This research was supported by NSF through grants ATM-0077555 and ATM-0417839, the DoD MURI program grant F4960-01-1-0359, and NASA grant NNG04GK18G. We would like to thank Jeffery P. Thayer for many helpful discussions regarding this work.

[60] Wolfgang Baumjohann thanks Stephan Buchert and two other reviewers for their assistance in evaluating this paper.

\section{References}

Banks, P. M., J. C. Foster, and J. Doupnik (1981), Chatanika radar observations relating to the latitudinal and local time variations of Joule heating, J. Geophys. Res., 86, 6869.

Codrescu, M. V., T. J. Fuller-Rowell, and J. C. Foster (1995), On the importance of $E$-field variability for Joule heating in the high-latitude thermosphere, Geophys. Res. Lett., 22, 2393.

Codrescu, M. V., T. J. Fuller-Rowell, J. C. Foster, J. M. Holt, and S. J. Cariglia (2000), Electric field variability associated with the Millstone Hill electric field model, J. Geophys. Res., 105, 5265.

Colegrove, F. D., F. S. Johnson, and W. Hanson (1966), Atmospheric composition in the lower thermosphere, J. Geophys. Res., 71, 2227.

Crowley, G., and C. L. Hackert (2001), Quantification of high latitude electric field variability, Geophys. Res. Lett., 28, 2783.

DeJong, A. D., and C. R. Clauer (2005), Polar UVI images to study steady magnetospheric convection events: Initial results, Geophys. Res. Lett., 32 , L24101, doi:10.1029/2005GL024498.

de La Beaujardiére, O., R. Johnson, and V. B. Wickwar (1991), Groundbased measurements of Joule heating rates, in Auroral Physics, edited by C.-I. Meng, M. J. Rycroft, and L. A. Frank, p. 439, Cambridge Univ. Press, New York.

Deng, Y., and A. J. Ridley (2006a), Dependence of neutral winds on convection $E$ field, solar EUV and auroral particle precipitation at high latitudes, J. Geophys. Res., 111, A09306, doi:10.1029/2005JA011368. 
Deng, Y., and A. J. Ridley (2006b), The role of vertical ion convection in the high-latitude ionospheric plasma distribution, J. Geophys. Res., 111, A09314, doi:10.1029/2006JA011637.

Emery, B. A., C. Lathuillere, P. G. Richards, R. G. Roble, M. J. Buonsanto, D. J. Knipp, P. Wilkinson, D. P. Sipler, and R. Niciejewski (1999), Time dependent thermospheric neutral response tothe 2-11 November 1993 storm period, J. Atmos. Terr. Phys., 61, 329-350.

Foster, J. C. (1983), An empirical electric field model derived from Chatanika radar data, J. Geophys. Res., 90, 981.

Fuller-Rowell, T. J., and D. Evans (1987), Height-integrated Pedersen and Hall conductivity patterns inferred from TIROS-NOAA satellite data, J. Geophys. Res., 92, 7606.

Fuller-Rowell, T. J., M. V. Codrescu, R. J. Moffett, and S. Quegan (1994), Response of the thermosphere and ionosphere to geomagnetic storms, J. Geophys. Res., 99, 3893.

Gary, J. B., R. A. Heelis, and J. P. Thayer (1995), Summary of field-aligned poynting flux observations from DE 2, Geophys. Res. Lett., 22, 1861.

Hardy, D. A., M. S. Gussenhoven, R. Raistrick, and W. J. McNeil (1987), Statistical and fuctional representation of the pattern of auroral energy flux, number flux, and conductivity, J. Geophys. Res., 92, 12,275.

Hedin, A. E. (1983), A revised thermospheric model based on mass spectometer and incoherent scatter data: MSIS-83, J. Geophys. Res., 88, 10,170 .

Heelis, R. A., and W. R. Coley (1988), Global and local Joule heating effects seen by DE 2, J. Geophys. Res., 93, 7551.

Heppner, J. P., and N. C. Maynard (1987), Empirical high-latitude electric field models, J. Geophys. Res., 92, 4467.

Johnson, E. S., and R. A. Heelis (2005), Characteristics of ion velocity structure at high latitudes during steady southward interplanetary magnetic field conditions, J. Geophys. Res., 110, A12301, doi:10.1029 2005JA011130

Kamide, Y., and H. W. Kroehl (1987), A concise review of the utility of ground-based magnetic recordings for estimating the Joule heat production rate, Ann. Geophys., 5, 535.

Kelley, M. C., D. J. Knudsen, and J. F. Vickrey (1991), Poynting flux measurements on a satellite: A diagnostic tool for space research, J. Geophys. Res., 96, 201

Killeen, T. L., and R. G. Roble (1984), An analysis of the high-latitude thermospheric wind pattern calculated by a thermospheric general circulation model: 1. Momentum forcing, J. Geophys. Res., 89, 7509.

Kivanc, ö., and R. A. Heelis (1998), Spatial distribution of ionospheric plasma and field structures in the high-latitude $\mathrm{F}$ region, J. Geophys. Res., 103, 6955.

Liu, H., and H. Lühr (2005), Strong disturbance of the upper thermospheric density due to magnetic storms: CHAMP observations, J. Geophys. Res., 110, A09S29, doi:10.1029/2004JA010908.

Lu, G., A. D. Richmond, B. A. Emery, and R. G. Roble (1995a), Magnetosphere-ionosphere-thermosphere coupling: Effect of neutral winds on energy transfer and field-aligned current, J. Geophys. Res., 100, 19,643.

Lu, G., et al. (1995b), Characteristics of ionospheric convection and fieldaligned current in the dayside cusp region, J. Geophys. Res., 100, 11,845.

Lu, G., et al. (1998), Global energy deposition during the January 1997 ISTP event, J. Geophys. Res., 103, 11,685.

Lühr, H., M. Rother, W. Köhler, P. Ritter, and L. Grunwaldt (2004), Thermospheric up-welling in the cusp region: Evidence from CHAMP observations, Geophys. Res. Lett., 31, L06805, doi:10.1029/2003GL019314.

Lummerzheim, D., L. Peticolas, A. Otto, J. Styers, B. Lanchester, B. Bristow, and M. Conde (2004), Ionospheric heating in aurora: Observations, Eos Trans. $A G U, 85(47)$, Fall Meet. Suppl., Abstract SA13B-06.

Matsuo, T., A. D. Richmond, and K. Hensel (2003), High-latitude ionospheric electric field variability and electric potential derived from DE-2 plasma drift measurements: Dependence on IMF and dipole tilt, J. Geophys. Res., 108(A1), 1005, doi:10.1029/2002JA009429.

McHarg, M., F. Chun, D. Knipp, G. Lu, B. Emery, and A. Ridley (2005), High-latitude Joule heating response to IMF inputs, J. Geophys. Res., 110, A08309, doi:10.1029/2004JA010949.

Oyama, S., B. J. Watkins, S. Nozawa, S. Maeda, and M. Conde (2005), Vertical ion motion observed with incoherent scatter radars in the polar lower ionosphere, J. Geophys. Res., 110, A04302, doi:10.1029/ 2004JA010705

Powell, K. G., P. L. Roe, T. J. Linde, T. I. Gombosi, and D. L. D. Zeeuw (1999), A solution-adaptive upwind scheme for ideal magnetohydrodynamics, J. Comput. Phys., 154, 284.
Prölss, G. W., L. H. Brace, H. G. Mayr, G. R. Carignan, T. L. Killeen, and J. A. Klobuchar (1991), Ionospheric storm effects at subauroral latitudes: A case study, J. Geophys. Res., 96, 1275.

Rawer, K., D. Bilitza, and S. Ramakrishnan (1978), Goals and status of the international reference ionosphere, Rev. Geophys., 16, 177.

Rees, M. H. (1989), Physics and Chemistry of the Upper Atmosphere, Cambridge Univ. Press, New York.

Rich, F. J., M. S. Gussenhoven, and M. E. Greenspan (1987), Using simultaneous particle and field observations on a low altitude satellite to estimate Joule heat energy flow into the high latitude ionosphere, Ann. Geophys., 5, 527

Richmond, A. D. (1992), Assimilative mapping of ionospheric electrodynamics, Adv. Space Res., 12, 59.

Richmond, A. D. (1995), Ionospheric electrodynamics using magnetic apex coordinates, J. Geomagn. Geoelectr., 47, 191.

Richmond, A. D., and Y. Kamide (1988), Mapping electrodynamic features of the high-latitude ionosphere from localized observations: Technique, J. Geophys. Res., 93, 5741.

Richmond, A. D., C. Lathuillère, and S. Vennerstroem (2003), Winds in the high-latitude lower thermosphere: Dependence on the interplanetary magnetic field, J. Geophys. Res., 108(A2), 1066, doi:10.1029/ 2002JA009493.

Ridley, A. J., C. R. Clauer, G. Lu, and V. O. Papitashvili (1997), Ionospheric convection during nonsteady interplanetary magnetic field conditions, J. Geophys. Res., 102, 14,563.

Ridley, A. J., G. Crowley, and C. Freitas (2000), A statistical model of the ionospheric electric potential, Geophys. Res. Lett., 27, 3675.

Ridley, A. J., T. I. Gombosi, D. L. D. Zeeuw, C. R. Clauer, and A. D. Richmond (2003), Ionospheric control of the magnetospheric configuration: Neutral winds, J. Geophys. Res., 108(A8), 1328, doi:10.1029/ 2002JA009464

Ridley, A. J., Y. Deng, and G. Toth (2006), The global ionosphere-thermosphere model, J. Atmos. Sol. Terr. Phys., 68, 839.

Roble, R. G., R. E. Dickinson, and E. C. Ridley (1982), Global circulation and termperature structure of the thermosphere with high-latitude plasma convection, J. Geophys. Res., 87, 1599.

Roble, R. G., E. C. Ridley, A. D. Richmond, and R. E. Dickinson (1988), A coupled thermosphere/ionosphere general circulation model, Geophys. Res. Lett., 15, 1325 .

Schunk, R. W., and A. F. Nagy (1978), Electron temperatures in the F-region of the ionosphere, Rev. Geophys., 16, 355.

Schunk, R. W., and A. F. Nagy (2000), Ionospheres, Cambridge Univ. Press, New York.

Sharber, J. R., et al. (1988), UARS particle environment monitor observations during the November 1993 storm: Auroral morphology, spectral characterization and energy deposition, J. Geophys. Res., 103, 26,307.

Sojka, J. J., and R. W. Schunk (1989), Theoretical study of the seasonal behavior of the global ionosphere at solar maximum, J. Geophys. Res., 94, 6739

Styers, J. M., A. Otto, D. Lummerzheim, B. Bristow, and B. Lanchester (2004), Joule heating in small-scale aurora: Modeling, Eos Trans. AGU, 85(47), Fall Meet. Suppl., Abstract SA23A-0389.

Thayer, J. P. (1998), Height-resolved Joule heating rates in the high-latitude E region and the influence of neutral winds, J. Geophys. Res., 103, 471. Thayer, J. P., and J. Semeter (2004), The convergence of magnetospheric energy flux in the polar atmosphere, J. Atmos. Terr. Phys., 66, 807-824.

Thayer, J. P., J. F. Vickrey, R. A. Heelis, and J. B. Gary (1995), Interpretation and modeling of the high-latitude electromagnetic energy flux, J. Geophys. Res., 100, 19,715.

Tóth, G., et al. (2005), Space weather modeling framework: A new tool for the space science community, J. Geophys. Res., 110, A12226, doi:10.1029/2005JA011126

Weimer, D. R. (1996), A flexible, IMF dependent model of high-latitude electric potential having "space weather" applications, Geophys. Res. Lett., 23, 2549

Wescott, E. M., H. Stenbaek-Nielsen, M. Conde, M. Larsen, and D. Lummerzheim (2006), The HEX experiment: Determination of the neutral wind field from 120 to $185 \mathrm{~km}$ altitude near a stable premidnight auroral arc by triangulating the drift of rocket-deployed chemical trails, J. Geophys. Res., 111, A09302, doi:10.1029/2005JA011002.

Y. Deng, High Altitude Observatory, National Center for Atmospheric Research, Boulder, CO, USA. (ydeng@ucar.edu)

A. J. Ridley, Center for Space Environment Modeling, University of Michigan, Ann Arbor, MI, USA. (ridley@umich.edu) 